\title{
Cosmic rays from leptophilic dark matter decay via kinetic mixing
}

\author{
Alejandro Ibarra, ${ }^{a}$ Andreas Ringwald, ${ }^{b}$ David $\operatorname{Tran}^{a}$ and \\ Christoph Weniger ${ }^{b}$ \\ ${ }^{a}$ Physik-Department T30d, Technische Universität München, \\ James-Franck-Straße, 85748 Garching, Germany \\ ${ }^{b}$ Deutsches Elektronen-Synchrotron DESY, \\ Notkestraße 85, 22607 Hamburg, Germany. \\ E-mail: alejandro.ibarra@ph.tum.de, andreas.ringwald@desy.de, \\ david.tran@ph.tum.de, christoph.weniger@desy.de
}

Received April 6, 2009

Accepted July 1, 2009

Published August 14, 2009

\begin{abstract}
If interpreted in terms of decaying dark matter, the steep rise in the positron fraction of cosmic rays above $10 \mathrm{GeV}$, as observed by the PAMELA experiment, suggests an underlying production mechanism that favors leptonic channels. We consider a scenario where a portion of the dark matter is made of the gauginos of an unbroken hidden-sector $\mathrm{U}(1)_{\mathrm{X}}$, which interact with the visible sector only through a tiny kinetic mixing. The second component of the dark matter is made of neutralinos, and depending on the mass spectrum, either the lightest neutralino or the hidden gaugino becomes unstable and subject to decay. We analyze the cosmic rays, namely the contributions to the positron, the extragalactic gamma-ray and the antiproton flux, which potentially result from these decays and demonstrate that the production of antiprotons can be naturally suppressed. Furthermore, we briefly discuss the apparent double-peak structure of the ATIC data in light of cascade-decaying hidden gauginos, as well as possible signatures at Fermi.
\end{abstract}

Keywords: cosmic ray theory, supersymmetry and cosmology, dark matter theory

ArXiv ePrint: 0903.3625 


\section{Contents}

1 Introduction 1

2 Model 2

3 Propagation models $\quad 5$

4 Results $\quad 10$

$\begin{array}{lll}4.1 & \text { Decaying neutralinos } & 10\end{array}$

$\begin{array}{lll}4.2 & \text { Decaying hidden gauginos } & 14\end{array}$

5 Conclusions $\quad 18$

$\begin{array}{ll}\text { A Implications of recent Fermi LAT results } & 19\end{array}$

\section{Introduction}

The PAMELA collaboration has recently reported a measurement of the positron fraction [1] which shows a significant excess at high energies compared to the expectations from spallation of cosmic rays on the interstellar medium [2]. This result confirms hints at an excess in the positron fraction reported in the past by several experiments: HEAT 94/95/00 [3], CAPRICE94 [4] and AMS-01 [5]. More importantly, the PAMELA collaboration has provided an accurate measurement of the energy spectrum of the positron fraction, revealing a steep rise between 7 and $100 \mathrm{GeV}$, possibly extending to even higher energies.

The excess in the positron fraction has been interpreted as an indication for the annihilation [6-9] or the decay [10-12] of dark matter particles. If interpreted as dark matter annihilations, the dark matter particles must annihilate into $W, Z, e, \mu, \tau$ for any dark matter mass, or into $q, b, t, h$ for a multi-TeV dark matter mass. In both cases one needs large boost factors, e.g. from dark matter substructures, since the required annihilation rates are much larger than the cross-section suggested by cosmology, $\langle\sigma v\rangle \approx 3 \times 10^{-26} \mathrm{~cm}^{3} \mathrm{~s}$ [13], would lead one to expect. On the other hand, if interpreted as dark matter decay, the dark matter particles must have a mass larger than $\sim 300 \mathrm{GeV}$, a lifetime around $10^{26} \mathrm{~s}$, and the decay must proceed preferentially into charged leptons of the first and second generation [14]. The properties of the dark matter particles are also subject to constraints from the diffuse gamma-ray flux as measured by the EGRET instrument [15], from H.E.S.S. and VERITAS observations of dwarf galaxies [16], and the antiproton flux, as measured by PAMELA [17], BESS95 [18], BESS95/97 [19], CAPRICE94 [20], CAPRICE98 [21] and IMAX [22]. More concretely, the good agreement of the theoretical predictions for the antiproton flux with the measurements suggests that the dark matter particles annihilate or decay mostly into leptons. Moreover, if one additionally takes into account the spectral feature in the total electron+positron flux observed by the ATIC experiment [23], dark matter particle masses 
above $1 \mathrm{TeV}$ are suggested. ${ }^{1}$ Although the interpretation of the positron excess in terms of dark matter is very suggestive, it should be borne in mind that nearby astrophysical sources such as pulsars might produce sizable positron fluxes in the energy range explored by PAMELA [29].

In this paper we will discuss the cosmic-ray signatures of supersymmetric scenarios with a gaugino of an unbroken hidden-sector $\mathrm{U}(1)$ gauge group which interacts with the visible sector only via a tiny kinetic mixing [30]. We will speculate that the tiny kinetic mixing induces the decay of the dark matter particles into lighter supersymmetric particles and positrons, thus providing a potential explanation of the excess observed by PAMELA. Assuming exact $R$-parity conservation, two possibilities may arise. First, we will study the case that the hidden gaugino mass is smaller than the lightest neutralino mass. We will show that the neutralino mostly decays into two charged leptons and the hidden gaugino. The hard positrons produced in the decay can then potentially explain the steep rise in the positron fraction observed by PAMELA and the absence of an excess in the antiproton flux. Secondly, we will study the case that the lightest neutralino mass is smaller than the hidden gaugino mass. If this is the case, it is the hidden gaugino which decays into the lightest neutralino, either directly or in a cascade decay, when there are supersymmetric particles with masses between the hidden gaugino mass and the lightest neutralino mass. Either particle could be the dominant component of dark matter. However, we will focus in this work on the possibility that the dominant component of dark matter is the lightest neutralino, which may allow direct dark matter detection.

In section 2 we will briefly review the main features and possible origins of scenarios with a hidden Abelian gauge group and kinetic mixing. In section 3 we will review the procedure to calculate the gamma-ray, positron and antiproton fluxes at Earth from dark matter decay. In section 4 we will present our results for the decaying neutralino and the decaying hidden gaugino case. Lastly, in section 5 we will present our conclusions.

\section{Model}

Many extensions of the Minimal Supersymmetric Standard Model (MSSM) contemplate the possibility of a hidden sector, consisting of superfields which are singlets under the Standard Model gauge group. Hidden-sector superfields usually couple very weakly to our observable sector, thus constituting a very natural arena for finding dark matter candidates. We consider an extension of the MSSM by a hidden Abelian gauge group $\mathrm{U}(1)_{\mathrm{X}}$ (for details about the model see ref. [30]). This gauge group remains unbroken at low energies and couples to the MSSM only through a tiny kinetic mixing, $\chi$, with the hypercharge $\mathrm{U}(1)_{\mathrm{Y}}[31]$ :

$$
\mathrm{SU}(3)_{\mathrm{c}} \times \mathrm{SU}(2) \times \underbrace{\mathrm{U}(1)_{\mathrm{Y}} \times \mathrm{U}(1)_{\mathrm{X}}}_{\text {kin. mixing } \chi} .
$$

We assume that all matter states charged under $\mathrm{U}(1)_{\mathrm{X}}$ are heavy and negligible. As a consequence the $\mathrm{U}(1)_{\mathrm{X}}$ gauge boson completely decouples from the observable sector. However, as shown in ref. [30], a non-zero mass-mixing of the order of $\delta M \sim \mathcal{O}\left(\chi \cdot M_{X}\right)$ between hidden gaugino and bino generally remains. More precisely, in the basis where the kinetic terms are

\footnotetext{
${ }^{1}$ For the combined electron+positron flux, we use results from measurements that have been undertaken by ATIC, HEAT94/95 [24], PPB-BETS [25], H.E.S.S. [26], BETS [27], CAPRICE94 [4] and SANRIKU [28].
} 
canonical, the extended $(5 \times 5)$ neutralino mass matrix reads, to lowest order in $\chi$,

$$
\mathcal{M}_{\mathrm{N}}=\left(\begin{array}{ccccc}
M_{X} & \delta M & 0 & 0 & 0 \\
\delta M & M_{1} & 0 & -M_{Z} c_{\beta} s_{W} & M_{Z} s_{\beta} s_{W} \\
0 & 0 & M_{2} & M_{Z} c_{\beta} c_{W} & -M_{Z} s_{\beta} c_{W} \\
0 & -M_{Z} c_{\beta} s_{W} & M_{Z} c_{\beta} c_{W} & 0 & -\mu \\
0 & M_{Z} s_{\beta} s_{W} & -M_{Z} s_{\beta} c_{W} & -\mu & 0
\end{array}\right) .
$$

Here, $\mu$ denotes the MSSM $\mu$-term, $M_{Z}$ the mass of the $Z^{0}$ gauge boson, $M_{1}$ and $M_{2}$ the bino and wino masses, respectively, $s_{W}$ the sine of the Weinberg angle and $s_{\beta}$ is related to the ratio of the two Higgs VEVs.

Below, we will concentrate on the case where the lightest supersymmetric particle in the visible sector is a bino-like neutralino $\chi_{1}^{0}$. Then, depending on the masses of the hidden gaugino and of the neutralino, one of the two particles becomes unstable with a lifetime that is roughly given by

$$
\tau_{X, \chi_{1}^{0}} \sim \mathcal{O}\left(10^{-2}-10\right) \times 10^{26} \mathrm{~s} \cdot\left(\frac{M_{X, \chi_{1}^{0}}}{100 \mathrm{GeV}}\right)^{-1}\left(\frac{\theta}{10^{-24}}\right)^{-2},
$$

where we made use of the mixing angle $\theta \simeq \delta M /\left|M_{\chi_{1}^{0}}-M_{X}\right| \sim \mathcal{O}(\chi)$. The exact prefactor depends on the dominant decay modes and on the mass spectrum of the supersymmetric particles. However, it is apparent that a lifetime around $10^{26} \mathrm{~s}$, as required to fit the PAMELA excess with decaying dark matter particles, implies an extremely small mixing of the order of $\chi \sim 10^{-24}$. Note that the mixing must be somewhat larger when the decaying particle is only a subdominant component of the dark matter, but an upper bound of roughly $\sim 10^{-20}$ holds from the requirement that the particle relic abundance has not already decayed.

The thermal production of hidden gauginos by oscillations between bino and hidden gaugino, which generally takes place in the primeval MSSM plasma, is irrelevant for the mixing parameters that we are looking at [30]. However, the hidden gaugino may be produced non-thermally, e.g. in the decay of a heavy gravitino, or it may be a thermal relic of the hidden sector [32]. In the latter case one requires additional particles that are charged under the hidden $\mathrm{U}(1)_{\mathrm{X}}$ with masses around $M_{X}$, however. For simplicity, we will assume throughout this work that the lightest neutralino in the visible sector has the right energy density to make up the dominant part of the observed dark matter, whereas the abundance of the hidden gaugino is always subdominant, $\rho_{X} \ll \rho_{\chi_{1}^{0}} \simeq \rho_{\text {DM }}$.

The actual mass scale of the hidden gaugino depends on how the breaking of supersymmetry is mediated to the visible sector and the hidden $\mathrm{U}(1)_{\mathrm{X}} \mathrm{X}$. If the soft masses $\sim M_{\text {soft }}$ in the visible sector arise from gauge mediation, whereas $\mathrm{U}(1)_{\mathrm{X}}$ couples to the supersymmetrybreaking sector only gravitationally, the predicted mass hierarchy is $M_{\text {soft }} \gg m_{3 / 2} \sim M_{X}$, where $m_{3 / 2}$ denotes the mass of the gravitino. In this scenario the lightest neutralino cannot be dark matter, because it would decay into the gravitino in the early Universe. However, if we suppose gravity mediation to the visible sector and anomaly mediation [33] to the $\mathrm{U}(1)_{\mathrm{X}}$, one expects $M_{\text {soft }} \sim m_{3 / 2} \gg M_{X}$. If the soft masses of both the visible sector and the $\mathrm{U}(1)_{\mathrm{X}}$ arise from gravity mediation, one in general expects that all masses are of the same order $M_{\text {soft }} \sim m_{3 / 2} \sim M_{X}$. Below we will assume that the gravitino is heavy enough to have no impact on the decay modes, $m_{3 / 2}>\max \left(M_{X}, M_{\chi_{1}^{0}}\right)$.

Additional hidden sector U(1) gauge factors are a generic feature of string compactifications. For example, in the "mini-landscape" of orbifold compactifications of the heterotic 
string [34] one encounters, at the compactification scale, a breaking of the gauge symmetry to a theory involving many hidden $\mathrm{U}(1) \mathrm{s}$, e.g. $\mathrm{E}_{8} \times \mathrm{E}_{8} \rightarrow \mathrm{G}_{\mathrm{SM}} \times \mathrm{U}(1)^{4} \times\left[\mathrm{SO}(8) \times \mathrm{SU}(2) \times \mathrm{U}(1)^{3}\right]$ and the like. Similarly, type II compactifications generically invoke hidden sector U(1)s, often also for global consistency requirements. Some of these hidden U(1)s may remain unbroken down to very small scales [35].

Kinetic mixing is generated by the exchange of heavy messengers that couple both to the hypercharge $\mathrm{U}(1)_{\mathrm{Y}}$ as well as to the hidden $\mathrm{U}(1)_{\mathrm{X}}$. Correspondingly, it is loopsuppressed, $\chi=g_{Y} g_{X} C /\left(16 \pi^{2}\right)$, where $g_{Y}$ and $g_{X}$ are the Abelian gauge couplings and $C$ is a dimensionless constant. In field-theoretic setups, the latter is naturally of order one [31] and thus way too large for our purposes. However, it can be much smaller if there are additional gauge or global symmetries (cf. refs. [30, 36]). Moreover, in models arising from string compactifications rather small mixings seem to be generic [35, 37-39]. In the context of compactifications of the heterotic string, the size of the mixing has been estimated as [37]

$$
\chi \sim \frac{g_{Y} g_{X}}{16 \pi^{2}} \frac{\Delta m}{M_{P}},
$$

where $\Delta m \ll M_{P}$ is the mass splitting in the messenger sector and $M_{P}$ is the Planck scale. Indeed, the mixing is quite small in this case (e.g. $\chi \sim 10^{-16}$, if $\Delta m$ is associated with gauge mediated supersymmetry breaking, $\Delta m \sim 100 \mathrm{TeV}$ ), but still to large for our purposes. However, a sufficiently strong suppression of the coupling could be achieved in models with multiple hidden U(1)s [30].

In compactifications of type-II string theories, where the hidden $\mathrm{U}(1) \mathrm{s}$ arise via D-branes in the (extra-dimensional) bulk that have no intersection with the branes responsible for the visible sector, one has to distinguish two cases.

i) In large volume scenarios, where the hidden D3-branes and the branes supporting hypercharge are located at generic positions in some Calabi-Yau, kinetic mixing is suppressed by the large volume $V_{6}$ of the extra dimensions [39],

$$
\chi \simeq \frac{g_{Y} g_{X}}{16 \pi^{2}}\left(V_{6} M_{s}^{6}\right)^{-2 / 3},
$$

where $M_{s}$ is the string scale, which is related to the Planck scale via $M_{s} \sim$ $g_{s} M_{P} / \sqrt{V_{6} M_{s}^{6}}$, where $g_{s}$ is the string coupling. We thus see that we may obtain a kinetic mixing of order $10^{-24}$, if we assume $V_{6} M_{s}^{6} \sim 10^{32}$, corresponding to a string scale of order $M_{s} \sim \mathrm{TeV}$. However, this case is problematic because the mass of the gravitino is of the order of meV [40], with impact on the stability of supersymmetric dark matter candidates like the neutralino or hidden gaugino.

ii) In scenarios with significant warping, such as KKLT [41], the standard model stack of branes, notably the brane featuring the hypercharge $\mathrm{U}(1)$, is placed at a special position - at the tip of a warped throat - while the hidden brane is separated from it by a distance $d$ along the throat. In this case kinetic mixing may be exponentially suppressed [39] like

$$
\chi \sim \frac{g_{Y} g_{X}}{16 \pi^{2}} e^{-m d},
$$

reminiscent of a "Yukawa-type" interaction. In fact, in warped compactifications the closed string fields that mediate the kinetic mixing, notably the NS-NS $B$ form and the $\mathrm{R}-\mathrm{R} C$ form fields, acquire masses,

$$
m \sim n M_{s} /\left(V_{3} M_{s}^{3}\right),
$$




\begin{tabular}{|c|c|}
\hline \multicolumn{2}{|c|}{ Neutralino / hidden gaugino decay modes } \\
\hline$M_{\chi_{1}^{0}}>M_{X}$ & $M_{\chi_{1}^{0}}<M_{X}$ \\
$\chi_{1}^{0} \rightarrow\left\{\begin{array}{l}f \tilde{f}_{L / R}^{*} \rightarrow f \bar{f} X \\
X h^{0} \\
X Z^{0}\end{array}\right.$ & $X \rightarrow\left\{\begin{array}{l}f \tilde{\bar{f}}_{L / R}^{(*)} \rightarrow f \bar{f} \chi_{i}^{0} \\
\chi_{i}^{0} h^{0} \\
\chi_{i}^{0} Z^{0} \\
\chi_{j}^{ \pm} W^{\mp}\end{array}\right.$ \\
\hline
\end{tabular}

Table 1. Dominant decay modes. Depending on the masses of the hidden gaugino, $M_{X}$, and the lightest neutralino, $M_{\chi_{1}^{0}}$, one of the two particles becomes unstable with a lifetime roughly given by eq. (2.3). Since the three-body decay into fermion pairs $f \bar{f}$ is mainly mediated by virtual sfermions, $\tilde{f}^{*}$, we show this explicitly. Furthermore, when a sfermion is lighter than the decaying particle, the corresponding three-body decay crosses over to a cascade decay. The subsequent decay and fragmentation of the Higgs and gauge bosons, charginos and neutralinos is not shown. Note that the letter $f$ represents any lepton or quark.

for some integer $n$, from the vacuum expectation values of their three-form field strengths, $H_{3}=d B_{2}, F_{3}=d C_{2}$, which are threading three-cycles of volume $V_{3}$ and cause the warping. Therefore, we conclude that no strong fine-tuning is required to obtain a tiny kinetic mixing in a KKLT-like scenario. Indeed, the estimate (2.6) yields the desired small value $\chi \sim 10^{-24}$ for $m d \sim 48$.

As mentioned above, depending on the mass spectrum of the supersymmetric particles, either the lightest neutralino, $\chi_{1}^{0}$, or the hidden gaugino, $X$, becomes unstable. The relevant decay modes are shown in table 1 . These decays may be detectable as anomalous contributions to the cosmic-ray fluxes observed at Earth. We will discuss this in some detail in the next section.

\section{Propagation models}

The decay of dark matter particles induced by the kinetic mixing discussed above will generate potentially sizable numbers of Standard Model particles which may be detectable in cosmicray experiments if the dark matter decay rate is high enough. In the following, we will consider the cosmic-ray signatures resulting from the decay of neutralinos and hidden gauginos in three different channels, namely gamma rays, positrons and antiprotons. ${ }^{2}$ Interestingly, in all of these channels, experiments are now reaching sensitivities to dark matter lifetimes of the order of $10^{26} \mathrm{~s}$.

Gamma rays. Photons from the decay of $\mathrm{GeV}-\mathrm{TeV}$ mass dark matter particles will manifest themselves as an anomalous contribution to the diffuse extragalactic gamma-ray background. More specifically, there are two distinct contributions to the diffuse gamma-ray flux from dark matter decay. There is an anisotropic component from the decay of dark matter particles in the Milky Way halo, as well as an isotropic diffuse emission from dark matter decaying at cosmological distances. The latter contribution is red-shifted due to the

\footnotetext{
${ }^{2}$ Dark matter decay also produces a flux of neutrinos which are unfortunately unobservable due to the large atmospheric neutrino backgrounds [42].
} 
expansion of the Universe. Thus, for a dark matter mass $m_{\mathrm{DM}}$ and a dark matter lifetime of $\tau_{\mathrm{DM}}$, the gamma-ray flux will be given by the sum [43]

$$
\left[E^{2} \frac{d J}{d E}\right]_{\mathrm{DM}}=\left[E^{2} \frac{d J}{d E}\right]_{\text {halo }}+\left[E^{2} \frac{d J}{d E}\right]_{\mathrm{extra}}
$$

where the halo component is given by

$$
\left[E^{2} \frac{d J}{d E}\right]_{\text {halo }}=\frac{2 E^{2}}{m_{\mathrm{DM}}} \frac{d N_{\gamma}}{d E} \frac{1}{8 \pi \tau_{\mathrm{DM}}} \int_{\mathrm{los}} \rho_{\mathrm{DM}}(\vec{l}) d \vec{l},
$$

in which $\rho_{\mathrm{DM}}$ is the dark matter halo profile, which in the following we assume to be a Navarro-Frenk-White (NFW) [44] profile for definiteness. The results will have only a slight dependence on the particular choice of halo profile. $d N_{\gamma} / d E$ denotes the energy spectrum of photons from dark matter decay. The integration extends along the line of sight (los), yielding an angular dependence of the signal. For our results, we will average the anisotropic halo contribution over the whole sky excluding a band of $\pm 10^{\circ}$ around the Galactic disk. The extragalactic component is given by

$$
\left[E^{2} \frac{d J}{d E}\right]_{\mathrm{extra}}=\frac{2 E^{2}}{m_{\mathrm{DM}}} C_{\gamma} \int_{1}^{\infty} d y \frac{d N_{\gamma}}{d(y E)} \frac{y^{-3 / 2}}{\sqrt{1+\Omega_{\Lambda} / \Omega_{\mathrm{M}} y^{-3}}}
$$

with the coefficient

$$
C_{\gamma}=\frac{\Omega_{\mathrm{DM}} \rho_{\mathrm{c}}}{8 \pi \tau_{\mathrm{DM}} H_{0} \Omega_{\mathrm{M}}^{1 / 2}} .
$$

The integration is over the red-shift $z$, with $y=z+1$. In these expressions, $\Omega_{\Lambda} \simeq 3 \Omega_{\mathrm{M}}$, $\Omega_{\mathrm{M}} \simeq 0.24$ and $\Omega_{\mathrm{DM}} \simeq 0.20$ are the vacuum, matter and dark matter density parameters, respectively, while $H_{0}=70.1 \mathrm{~km} \mathrm{~s}^{-1} \mathrm{Mpc}^{-1}$ is the present value of the Hubble parameter and $\rho_{\mathrm{c}}$ is the critical density. One finds that the halo component is dominant, although both contributions are of the same order. For the background to the dark matter signal from conventional astrophysical sources, we will assume a power law spectrum that fits the data points below $1 \mathrm{GeV}$,

$$
\left[E^{2} \frac{d J}{d E}\right]_{\text {background }}=6.8 \times 10^{-7} E^{-0.32} \mathrm{GeV}\left(\mathrm{cm}^{2} \operatorname{str~s}\right)^{-1} .
$$

Antimatter propagation. At the energies of interest here, only antiparticles created within the Milky Way's dark matter halo will be of importance. Antimatter propagation in the Galaxy is a fairly complicated process due to diffusion, energy loss and annihilation effects. It is commonly described using a stationary two-zone diffusion model with cylindrical boundary conditions [45]. Under this approximation, the number density of antiparticles per unit kinetic energy, $f(T, \vec{r}, t)$, satisfies the following transport equation, which applies for both positrons and antiprotons:

$$
\frac{\partial f}{\partial t}=\nabla \cdot[K(T, \vec{r}) \nabla f]+\frac{\partial}{\partial T}[b(T, \vec{r}) f]-\nabla \cdot\left[\vec{V}_{c}(\vec{r}) f\right]-2 h \delta(z) \Gamma_{\text {ann }} f+Q(T, \vec{r}) .
$$

We assume free escape conditions for the solution $f(T, \vec{r}, t)$ at the boundary of the diffusion zone, which is approximated by a cylinder with half-height $L=1-15$ kpcand radius $R=$ $20 \mathrm{kpc}$, and solve the equation for the steady-state case, where $\partial f / \partial t=0$. 
The first term on the right-hand side of the transport equation is the diffusion term, which accounts for the propagation through the tangled Galactic magnetic field. The diffusion coefficient $K(T, \vec{r})$ is assumed to be constant throughout the diffusion zone and is parameterized by:

$$
K(T)=K_{0} \beta \mathcal{R}^{\delta},
$$

where $\beta=v / c$ is the velocity in units of the speed of light $c$, and $\mathcal{R}$ is the rigidity of the particle, which is defined as the momentum in GeV per unit charge, $\mathcal{R} \equiv p[\mathrm{GeV}] / Z$. The normalization $K_{0}$ and the spectral index $\delta$ of the diffusion coefficient are related to the properties of the interstellar medium and can be determined from flux measurements of other cosmicray species, mainly from the Boron-to-Carbon $(\mathrm{B} / \mathrm{C})$ ratio [46]. The second term accounts for energy losses due to inverse Compton scattering on starlight or the cosmic microwave background, synchrotron radiation and ionization. The third term is the convection term, which accounts for the drift of charged particles away from the disk induced by the Milky Way's Galactic wind. It has axial direction and is also assumed to be constant inside the diffusion region: $\vec{V}_{c}(\vec{r})=V_{c} \operatorname{sign}(z) \vec{k}$. The fourth term accounts for antimatter annihilation with rate $\Gamma_{\text {ann }}$, when it interacts with ordinary matter in the Galactic disk, which is assumed to be an "infinitely" thin disk with half-height $h=100$ pc. Note that, although this term is completely negligible in the case of positrons, it can have a rather small but noticeable effect on the number density of antiprotons. Lastly, $Q(T, \vec{r})$ is the source term of positrons or antiprotons which is given by

$$
Q(E, \vec{r})=\frac{\rho_{\mathrm{DM}}(\vec{r})}{m_{\mathrm{DM}} \tau_{\mathrm{DM}}} \frac{d N}{d E},
$$

where $d N / d E$ is the energy spectrum of antiparticles created via the decay of dark matter particles. In the transport equation, reacceleration effects and non-annihilating interactions of antimatter in the Galactic disk have been neglected.

The solution of the transport equation at the Solar System, $r=r_{\odot}, z=0$, can be formally expressed by the convolution

$$
f(T)=\frac{1}{m_{\mathrm{DM}} \tau_{\mathrm{DM}}} \int_{0}^{T \max } d T^{\prime} G\left(T, T^{\prime}\right) \frac{d N\left(T^{\prime}\right)}{d T^{\prime}},
$$

where $T_{\max }=m_{\mathrm{DM}}$ for the case of the positrons and $T_{\max }=m_{\mathrm{DM}}-m_{p}$ for the antiprotons. The solution is thus factorized into two parts. The first part, given by the Green's function $G\left(T, T^{\prime}\right)$, encodes all of the information about the astrophysics (such as the details of the halo profile and the propagation of antiparticles in the Galaxy). The remaining part depends exclusively on the nature and properties of the decaying dark matter particles, namely the mass, the lifetime and the energy spectrum of antiparticles produced in the decay.

We will consider the dark matter lifetime to be a free parameter that is constrained by requiring a qualitatively good agreement of the predicted positron fraction with the PAMELA results. Therefore, the only uncertainties in the computation of the antimatter fluxes stem from the determination of the Green's function, e.g. from the uncertainties in the propagation parameters and the halo profile. As it turns out, the uncertainties in the precise shape of the halo profile are not crucial for the determination of the primary antimatter fluxes, since the Earth receives mostly antimatter created within a few kpc from the Sun, where the different halo profiles are very similar. On the other hand, the uncertainties in the propagation parameters can substantially change the predictions for the antimatter fluxes, even by as much as two orders of magnitude for the antiproton flux. The reason for this 


\begin{tabular}{|c|ccc|}
\hline Model & $\delta$ & $K_{0}\left(\mathrm{kpc}^{2} / \mathrm{Myr}\right)$ & $L(\mathrm{kpc})$ \\
\hline M2 & 0.55 & 0.00595 & 1 \\
MED & 0.70 & 0.0112 & 4 \\
M1 & 0.46 & 0.0765 & 15 \\
\hline
\end{tabular}

Table 2. Astrophysical parameters compatible with the B/C ratio that yield the minimum (M2), median (MED) and maximal (M1) flux of positrons.

large uncertainty is a correlation among the diffusion parameters and the size of the diffusion zone. The ranges of the astrophysical parameters that are consistent with the $\mathrm{B} / \mathrm{C}$ ratio and that produce the maximal, median and minimal positron and antiproton fluxes are listed in table 2 and $3[47,48] .^{3}$

Positrons and antiprotons have different properties regarding their propagation, and their respective transport equations can be approximated by different limits of eq. (3.6). By exploiting the cylindrical symmetry of the problem, it is then possible to find semi-analytical solutions to the transport equation in each case. Approximate interpolating functions for the Green's function can be found in ref. [10].

Positron Flux. For the case of the positrons, Galactic convection and annihilations in the disk can be neglected in the transport equation, which is then simplified to:

$$
\nabla \cdot\left[K(T, \vec{r}) \nabla f_{e^{+}}\right]+\frac{\partial}{\partial T}\left[b(T, \vec{r}) f_{e^{+}}\right]+Q(T, \vec{r})=0,
$$

where the rate of energy loss, $b(T, \vec{r})$, is assumed to be a spatially constant function parameterized by $b(T)=\frac{T^{2}}{T_{0} \tau_{E}}$, with $T_{0}=1 \mathrm{GeV}$ and $\tau_{E}=10^{16} \mathrm{~s}$.

The solution to this equation is formally given by the convolution eq. (3.9). The explicit form of the Green's function is [8]

$$
G_{e^{+}}\left(T, T^{\prime}\right)=\sum_{n, m=1}^{\infty} B_{\mathrm{nm}}\left(T, T^{\prime}\right) J_{0}\left(\zeta_{n} \frac{r_{\odot}}{R}\right) \sin \left(\frac{m \pi}{2}\right),
$$

where $J_{0}$ is the zeroth-order Bessel function of the first kind, whose successive zeros are denoted by $\zeta_{n}$. On the other hand,

$$
B_{\mathrm{nm}}\left(T, T^{\prime}\right)=\frac{\tau_{E} T_{0}}{T^{2}} C_{\mathrm{nm}} \times \exp \left\{\left(\frac{\zeta_{n}^{2}}{R^{2}}+\frac{m^{2} \pi^{2}}{4 L^{2}}\right) \frac{K_{0} \tau_{E}}{\delta-1}\left[\left(\frac{T}{T_{0}}\right)^{\delta-1}-\left(\frac{T^{\prime}}{T_{0}}\right)^{\delta-1}\right]\right\},
$$

with

$$
C_{\mathrm{nm}}=\frac{2}{J_{1}^{2}\left(\zeta_{n}\right) R^{2} L} \int_{0}^{R} r^{\prime} d r^{\prime} \int_{-L}^{L} d z^{\prime} \rho\left(\vec{r}^{\prime}\right) J_{0}\left(\zeta_{n} \frac{r^{\prime}}{R}\right) \sin \left[\frac{m \pi}{2 L}\left(L-z^{\prime}\right)\right]
$$

where $J_{1}$ is the first-order Bessel function.

The interstellar positron flux from dark matter decay is finally given by

$$
\Phi_{e^{+}}^{\text {prim }}(T)=\frac{c}{4 \pi m_{\mathrm{DM}} \tau_{\mathrm{DM}}} \int_{0}^{m_{\mathrm{DM}}} d T^{\prime} G_{e^{+}}\left(T, T^{\prime}\right) \frac{d N_{e^{+}}\left(T^{\prime}\right)}{d T^{\prime}} .
$$

\footnotetext{
${ }^{3}$ In most cases, the propagated positron spectrum only depends mildly on the model that is actually used. For a comparison between different propagation models that feature smaller values of $\delta$ than the ones used in this paper, see e.g. ref. [49].
} 


\begin{tabular}{|c|cccc|}
\hline Model & $\delta$ & $K_{0}\left(\mathrm{kpc}^{2} / \mathrm{Myr}\right)$ & $L(\mathrm{kpc})$ & $V_{c}(\mathrm{~km} / \mathrm{s})$ \\
\hline MIN & 0.85 & 0.0016 & 1 & 13.5 \\
MED & 0.70 & 0.0112 & 4 & 12 \\
MAX & 0.46 & 0.0765 & 15 & 5 \\
\hline
\end{tabular}

Table 3. Astrophysical parameters compatible with the B/C ratio that yield the minimal (MIN), median (MED) and maximal (MAX) flux of antiprotons.

The dependence of the positron flux on the diffusion model is mostly important at low energies, where the signal lies well below the background.

Rather than measuring the positron flux, most experiments measure the positron fraction, $\Phi_{e^{+}} /\left(\Phi_{e^{-}}+\Phi_{e^{+}}\right)$, since most sources of systematic errors, such as detector acceptance or trigger efficiency, cancel out when computing the ratio of particle fluxes. The background to the positron flux from dark matter decay will be constituted by a secondary positron flux originating from the collision of primary protons and other nuclei on the interstellar medium.

For the background fluxes of primary and secondary electrons, as well as secondary positrons, we use the parameterizations obtained in ref. [7] from the GALPROP numerical code for cosmic-ray propagation [2]. We leave the normalization of the primary electron flux as a free parameter to be fitted in order to match the observations of the positron fraction.

Antiproton flux. The general transport equation, eq. (3.6), can be simplified by taking into account that energy losses are negligible for antiprotons. Therefore, the transport equation for the antiproton density, $f_{\bar{p}}(T, \vec{r}, t)$, is then given by:

$$
0=\frac{\partial f_{\bar{p}}}{\partial t}=\nabla \cdot\left(K(T, \vec{r}) \nabla f_{\bar{p}}\right)-\nabla \cdot\left(\vec{V}_{c}(\vec{r}) f_{\bar{p}}\right)-2 h \delta(z) \Gamma_{\text {ann }} f_{\bar{p}}+Q(T, \vec{r}),
$$

where the annihilation rate, $\Gamma_{\text {ann }}$, is determined by the Galactic Hydrogen and Helium densities and the proton-antiproton scattering cross-section, for which we use the parameterization by Tan and $\mathrm{Ng}[50]$.

Analogously to the positron case, the solution to the transport equation can be expressed as a convolution of the form eq. (3.9). The analytic expression for the Green's function reads [51]:

$$
G_{\bar{p}}\left(T, T^{\prime}\right)=\sum_{i=1}^{\infty} \exp \left(-\frac{V_{c} L}{2 K(T)}\right) \times \frac{y_{i}(T)}{A_{i}(T) \sinh \left(S_{i}(T) L / 2\right)} J_{0}\left(\zeta_{i} \frac{r_{\odot}}{R}\right) \delta\left(T-T^{\prime}\right),
$$

where

$$
y_{i}(T)=\frac{4}{J_{1}^{2}\left(\zeta_{i}\right) R^{2}} \int_{0}^{R} r^{\prime} d r^{\prime} J_{0}\left(\zeta_{i} \frac{r^{\prime}}{R}\right) \times \int_{0}^{L} d z^{\prime} \exp \left(\frac{V_{c}\left(L-z^{\prime}\right)}{2 K(T)}\right) \sinh \left(\frac{S_{i}\left(L-z^{\prime}\right)}{2}\right) \rho\left(\vec{r}^{\prime}\right),
$$

and

$$
\begin{aligned}
A_{i}(T) & =2 h \Gamma_{\mathrm{ann}}(T)+V_{c}+k S_{i}(T) \operatorname{coth} \frac{S_{i}(T) L}{2}, \\
S_{i}(T) & =\sqrt{\frac{V_{c}^{2}}{K(T)^{2}}+\frac{4 \zeta_{i}^{2}}{R^{2}}} .
\end{aligned}
$$


The interstellar antiproton flux is then given by

$$
\Phi_{\bar{p}}^{\mathrm{IS}}(T)=\frac{v_{\bar{p}}(T)}{4 \pi m_{\mathrm{DM}} \tau_{\mathrm{DM}}} \int_{0}^{m_{\mathrm{DM}}-m_{p}} d T^{\prime} G_{\bar{p}}\left(T, T^{\prime}\right) \frac{d N_{\bar{p}}\left(T^{\prime}\right)}{d T^{\prime}},
$$

where $v_{\bar{p}}(T)$ is the antiproton velocity. However, in order to compare the calculated antiproton spectrum with experimental results, one also has to take into account the effect of solar modulation. In the force-field approximation $[52,53]$ the effect of solar modulation can be included by applying the following relation between the antiproton flux at the top of the Earth's atmosphere and the interstellar antiproton flux [54]:

$$
\Phi_{\bar{p}}^{\mathrm{TOA}}\left(T_{\mathrm{TOA}}\right)=\left(\frac{2 m_{p} T_{\mathrm{TOA}}+T_{\mathrm{TOA}}^{2}}{2 m_{p} T_{\mathrm{IS}}+T_{\mathrm{IS}}^{2}}\right) \Phi_{\bar{p}}^{\mathrm{IS}}\left(T_{\mathrm{IS}}\right),
$$

where $T_{\mathrm{IS}}=T_{\mathrm{TOA}}+\phi_{F}$, with $T_{\mathrm{IS}}$ and $T_{\mathrm{TOA}}$ being the antiproton kinetic energies at the heliospheric boundary and at the top of the Earth's atmosphere, respectively, and $\phi_{F}$ being the solar modulation parameter, which varies between $500 \mathrm{MV}$ and 1.3 GV over the eleven-year solar cycle. Since experiments are usually undertaken near solar minimum activity, we will choose $\phi_{F}=500 \mathrm{MV}$ for our numerical analysis in order to compare our predicted flux with the collected data. For the antiprotons, we will not examine the background from spallation, but simply require that the fluxes from dark matter decay lie appropriately below the measurements so as to be compatible with predominantly secondary antiproton production.

\section{Results}

Below, we will present our results for the cosmic-ray signatures of decaying neutralinos and decaying hidden gauginos. ${ }^{4}$ In both cases, we will start with an analysis of the predictions that follow when assuming that the visible sector is described by an exemplary point in the coannihilation region of the mSUGRA parameter space. This ensures a consistent cosmology in the visible sector and that all free parameters of the MSSM are fixed. After that, we will go beyond this mSUGRA scenario and discuss how the cosmic-ray signatures can change in more generic cases. This will include a discussion about cascade decays in light of the apparent double-peak structure of the ATIC data.

\subsection{Decaying neutralinos}

The potentially relevant decay modes for the case $M_{X}<M_{\chi_{1}^{0}}$, where the lightest neutralino can decay into the hidden gaugino, are summarized in table 1 . Beside three body decays, which produce fermion/anti-fermion pairs, we also have to take into account the decay into Higgs and $Z^{0}$ bosons. Throughout the analysis we will assume that the lightest neutralino $\chi_{1}^{0}$ makes up the dominant part of the dark matter, $\rho_{\chi_{1}^{0}} \simeq \rho_{\mathrm{DM}}$.

mSUGRA point. As stated above, our exemplary mSUGRA model lies in the coannihilation region. The defining parameters are $m_{0}=150 \mathrm{GeV}, m_{1 / 2}=720 \mathrm{GeV}, A_{0}=0$, $\tan \beta=10$ and $\operatorname{sign} \mu=+1$. In this model the lightest neutralino has a mass of $301 \mathrm{GeV}$ and the correct relic density to be dark matter, $\Omega h^{2} \simeq 0.104 .{ }^{5}$ As typical for models in the coannihilation region, the three right-handed sleptons have masses around $304-307 \mathrm{GeV}$,

\footnotetext{
${ }^{4}$ For a short discussion of our model in light of the new Fermi electron data [55] see the appendix.

${ }^{5}$ The mass spectrum and relic abundance were calculated with the aid of DarkSUSY 5.0.4 [56].
} 


\begin{tabular}{|ccccccc|}
\hline \multirow{2}{*}{$M_{X}[\mathrm{GeV}]$} & \multicolumn{7}{c|}{ Branching Ratios for $\chi_{1}^{0} \rightarrow$} & \multirow{2}{*}[10^{26}\mathrm{s}]{} \\
\hline 1 & $28 \%$ & $28 \%$ & $32 \%$ & $8.8 \%$ & $2.6 \%$ & 1.8 \\
50 & $27 \%$ & $27 \%$ & $30 \%$ & $13 \%$ & $2.4 \%$ & 1.7 \\
100 & $24 \%$ & $24 \%$ & $28 \%$ & $21 \%$ & $2.4 \%$ & 1.5 \\
150 & $21 \%$ & $21 \%$ & $24 \%$ & $32 \%$ & $2.6 \%$ & 1.3 \\
200 & $30 \%$ & $30 \%$ & $36 \%$ & - & $3.7 \%$ & \\
\hline
\end{tabular}

Table 4. Branching ratios for the decay of a neutralino $\chi_{1}^{0}$ into a lighter hidden gaugino $X$, for different hidden gaugino masses $M_{X}$. In the visible sector, masses and mixing parameters are fixed by a mSUGRA scenario in the coannihilation region as described in the text. The lightest neutralino has a mass of $301 \mathrm{GeV}$. Branching ratios of three-body decays into neutrinos, $\chi_{1}^{0} \rightarrow \nu \bar{\nu} X$, and quarks, $\chi_{1}^{0} \rightarrow q \bar{q} X$, are smaller than $0.3 \%$ and $0.02 \%$, respectively. The two-body decay into photons, $\chi_{1}^{0} \rightarrow \gamma X$, is one-loop suppressed and neglected. We also indicate the lifetime of the neutralino which gives the best fit to the data.

which is similar to the mass of the lightest neutralino. The left-handed sleptons have masses around $500 \mathrm{GeV}$. Furthermore, this particular mSUGRA point, which features e.g. a spinindependent cross section per proton of $2.6 \times 10^{-46} \mathrm{~cm}^{2}$, can be probed with the next-to-next generation direct DM detection experiments like XENON1T and LUX/ZEP.

The dominant branching ratios of the neutralino decay are summarized in table 4 , for different masses of the hidden gaugino. ${ }^{6}$ Most interestingly, the fraction of decays into charged leptonic final states is never below $\sim 65 \%$. Beside the small masses of the righthanded sleptons, the underlying reason is the large $\mu$-term, $\mu=865 \mathrm{GeV}$, which suppresses the mixing between the bino-like lightest neutralino $\chi_{1}^{0}$, the hidden gaugino and the higgsinos like $\sim \mathcal{O}\left(M_{Z} / \mu\right)$. Furthermore, for large enough masses of the hidden gaugino, the decay into $h^{0} X$ becomes kinematically forbidden.

Note that although we present a concrete mSUGRA model for definiteness, the branching ratios shown here are typical for mSUGRA models in the coannihilation region, provided that the $\mu$-term is large enough. If the latter is the case, three-body decays into charged leptons dominate over two-body decays into Higgs and gauge bosons.

To obtain the energy distribution of gamma rays, positrons and antiprotons that are produced in the neutralino decay, we used the event generator PYTHIA 6.4 [59]. From these spectra, the contribution to cosmic-ray fluxes as measurable at Earth can be derived as described in the previous section. Note that the lifetime of the neutralino is always fixed by requiring a qualitatively good agreement with the positron fraction as measured by PAMELA.

Our results are shown in figure 1. We find that in principle the model can account for the observed excess in the positron fraction around 10-100 if the hidden gaugino is light with a mass $M_{X} \lesssim 50 \mathrm{GeV}$, although the predicted peak seems to rise too slowly to fully match the PAMELA data. This slow rise is due to the two-body decay into Higgs bosons, whose subsequent fragmentation produces rather soft positrons. From the lower plots of figure 1 it is apparent that the model is compatible with the EGRET measurements of the extragalactic gamma-ray background. ${ }^{7}$ However, the contribution to the antiproton flux can

\footnotetext{
${ }^{6}$ The calculations were done with FeynA rts 3.4 and FormCalc 5.4 [57, 58].

${ }^{7}$ Gamma rays with energies below $\sim 10 \mathrm{GeV}$ stem from the fragmentation of the Higgs boson whereas gamma rays at higher energies mainly come from $\tau$ decay.
} 

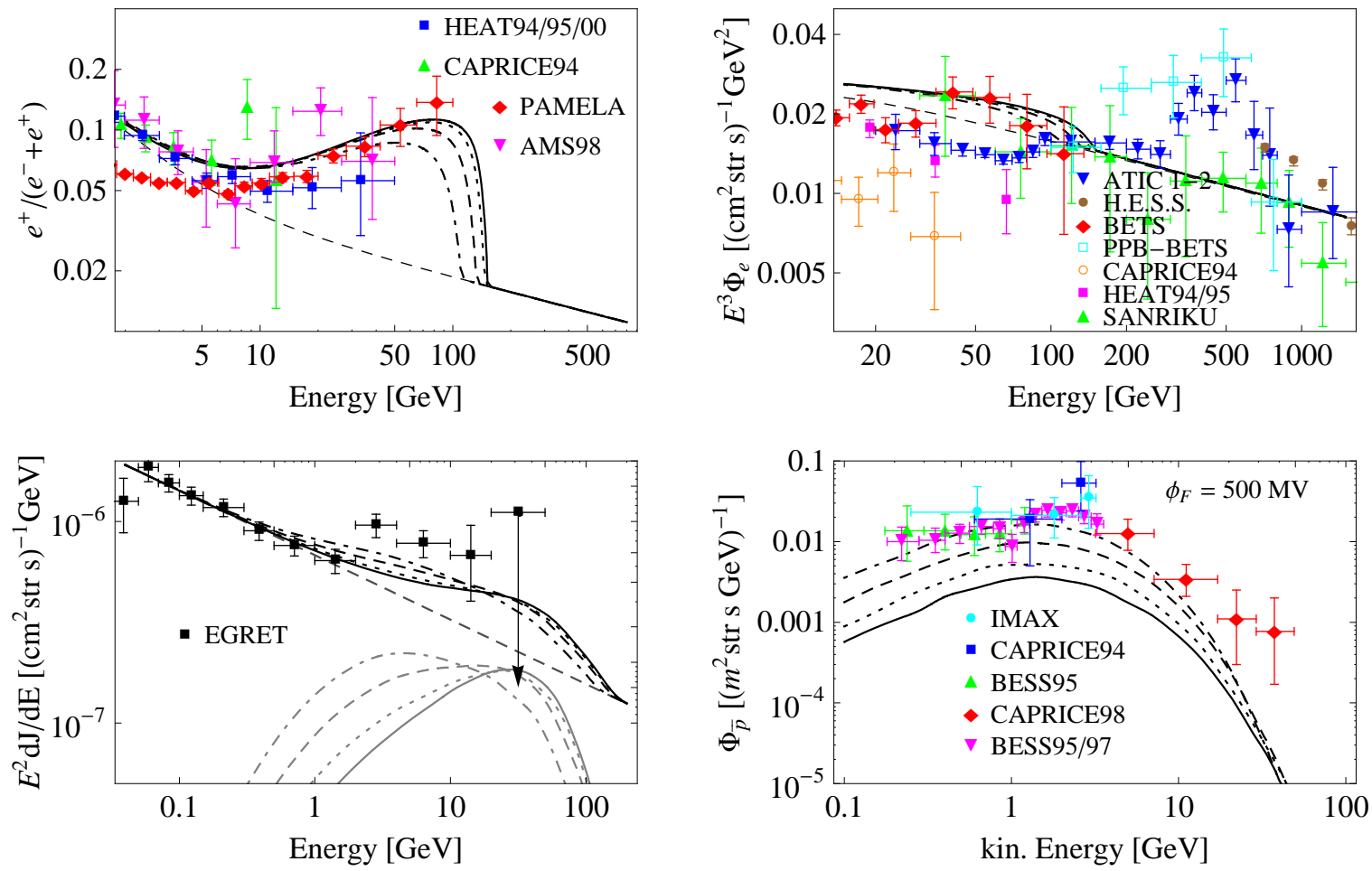

Figure 1. Positron fraction, total electron+positron, extragalactic gamma-ray and antiproton flux of a decaying neutralino $\chi_{1}^{0}$ as predicted for our exemplary mSUGRA scenario. The used branching ratios are shown in table 4 . The mass of the decaying neutralino is $301 \mathrm{GeV}$, the hidden gaugino mass varies between $1 \mathrm{GeV}$ (solid), $50 \mathrm{GeV}$ (dotted), $100 \mathrm{GeV}$ (dashed) and $150 \mathrm{GeV}$ (dot-dashed). We used the MED propagation model. In the lower left plot, the grey lines indicate the flux without background. In the lower right plot, we only show the flux without background.

be problematic for hidden gaugino masses above $\sim 100 \mathrm{GeV} .{ }^{8}$ Of course, the peak in the ATIC data around $300-800 \mathrm{GeV}$ cannot be reproduced in this setup. ${ }^{9}$

Idealized three-body decay of a heavy neutralino. For different parameters of the underlying MSSM model, the above plots can mainly change in two ways. Firstly, a larger value of the $\mu$-parameter would reduce the branching ratio into Higgs and $Z^{0}$ bosons. ${ }^{10}$ As a result, the rise in the positron fraction would be steeper, and the contribution to the

\footnotetext{
${ }^{8}$ Note that the uncertainty in the antiproton flux at Earth from dark matter decay can be as large as one order of magnitude in both directions [10], due to our ignorance of the precise propagation parameters.

${ }^{9}$ In figure 1 we aimed to fit the PAMELA data. Since we restricted ourself to use the background fluxes from ref. [7], as described above, this results in an electron+positron flux that lies above the ATIC data points at energies below $100 \mathrm{GeV}$. A lower normalization of the background flux of primary electrons would increase the agreement with ATIC, while at the same time flattening the positron fraction somewhat. Note that this behavior is common to all simultaneous fits to ATIC and PAMELA when using the above backgrounds. The same reasoning holds for figures 2,3 and 5 .

${ }^{10} \mathrm{~A}$ concrete lower bound on the $\mu$-parameter is extremely model-dependent. However, in the concrete scenario with a bino-like lightest neutralino where we take $\tan \beta=10, \alpha_{h}=-0.1$ and assume that the righthanded sleptons have a mass around $1.02 \cdot M_{\chi_{1}^{0}}$, the lower bound $\mu \gtrsim \mathcal{O}\left(2 M_{\chi_{1}^{0}}\right)$ turns out to be sufficient to suppress the branching ratio into Higgs bosons below $20 \%$.
} 

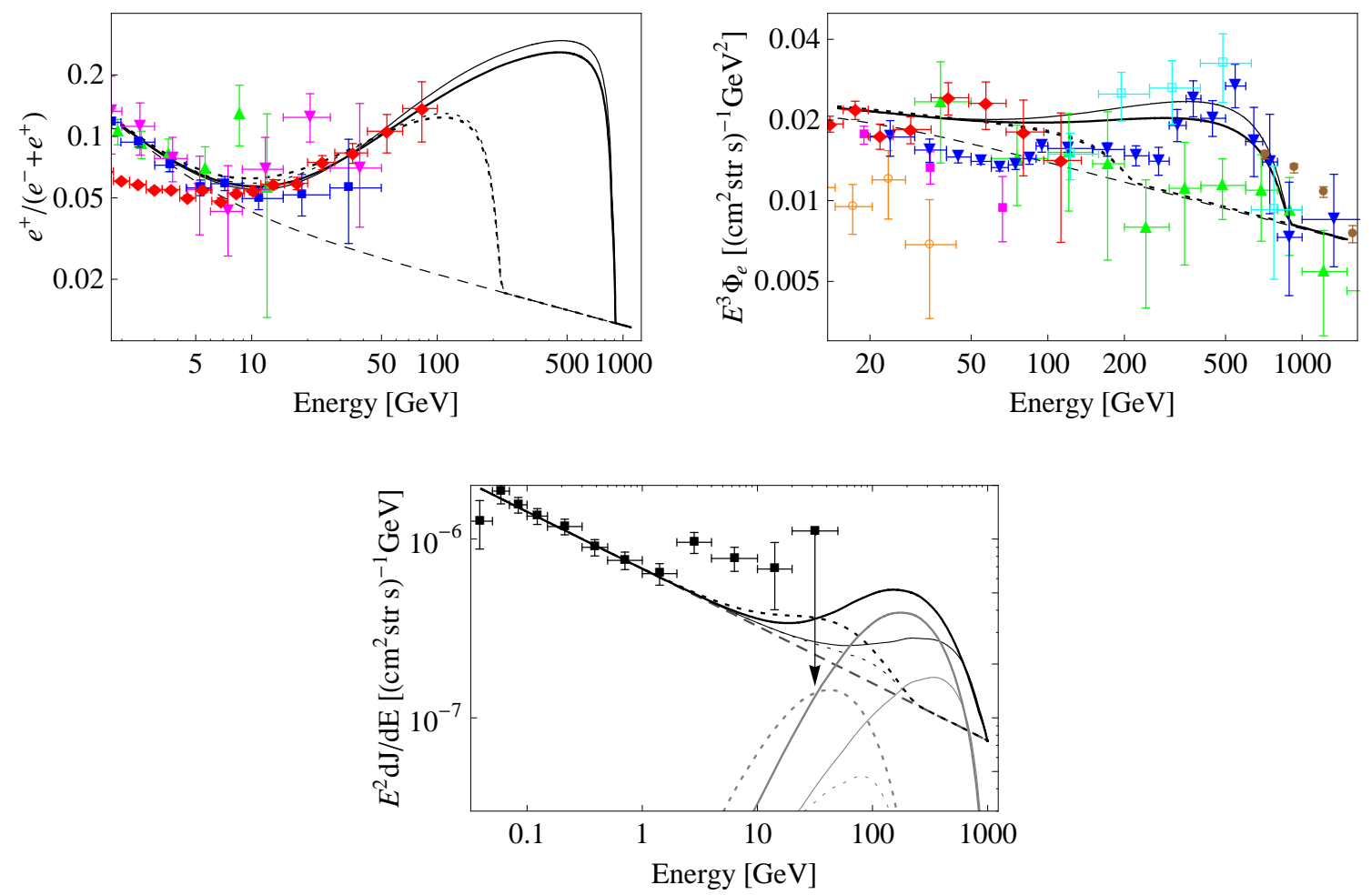

Figure 2. Positron fraction, total electron+positron flux and extragalactic gamma-ray flux for an idealized, three-body decaying bino-like neutralino. We neglect effects from $h^{0}$ and $Z^{0}$ bosons and assume pure democratic three-body decay into charged lepton pairs. The masses of the neutralino and the hidden gaugino are $500 \mathrm{GeV}$ and $150 \mathrm{GeV}$ (thick solid lines) or $1850 \mathrm{GeV}$ and $300 \mathrm{GeV}$ (thick dotted lines), respectively. The thin lines show the predictions when the decay into the tau-channel is neglected. The mass of the right-handed sleptons is assumed to be by a factor 1.1 larger than the neutralino mass.

antiproton flux smaller. Secondly, a higher mass of the decaying neutralino would shift the peak to higher energies, as suggested by the ATIC data.

In figure 2 we show our results for the cosmic-ray fluxes in the idealized case where a bino-like neutralino decays only via virtual right-handed sleptons. This resembles scenarios with a large $\mu$-term and large masses for the left-handed sleptons. The masses of the hidden gaugino and the neutralino $\chi_{1}^{0}$ are $150 \mathrm{GeV}$ and $500 \mathrm{GeV}$ (solid lines) or $300 \mathrm{GeV}$ and $1850 \mathrm{GeV}$ (dotted lines), respectively. Note that the thick lines correspond to the standard case where the neutralino decays democratically into all three flavors.

As expected, the rise in the positron fraction is now steeper and can easily accommodate the PAMELA data. Furthermore, a very heavy neutralino around $1.8 \mathrm{TeV}$ allows to also account for the ATIC excess. In any case we find a clear excess in the extragalactic gammaray flux at energies above $10 \mathrm{GeV}$.

The gamma rays come mainly from $\tau$ decays and bremsstrahlung, but the latter is a subdominant effect as long as the three-body decay into charged leptons is democratic. However, the decay into taus can be suppressed in cases where the stau mixing angle is large, since the correspondingly larger left-handed component of the lighter stau weakens the coupling to the bino-like neutralino and the hidden gaugino. For example, if the lighter stau is equally left- and right-handed, the three-body decay into taus would be suppressed 
by a factor of $\sim 0.5$. To obtain a lower bound on the predicted gamma-ray signal, we also show the case where the neutralino decays into the first two generations only (see thin lines in figure 2). The gamma-ray flux is much smaller in this case and comes mainly from bremsstrahlung of the electrons produced in the three-body decay $\chi_{1}^{0} \rightarrow e^{-} e^{+} X$. Note that in any case we obtain a tight correlation between the contributions to the positron flux and the extragalactic gamma-ray flux, where the latter comes mainly from $\tau$ decays in most cases.

Heavy bino-like neutralinos with masses above a few hundred $\mathrm{GeV}$ are problematic for cosmology since they are typically overproduced, even when coannihilation with sleptons is taken into account. At the same time, wino- and higgsino-like lightest neutralinos do not exhibit the desired leptophilic decay. ${ }^{11}$ However, these problems are absent if one considers scenarios where the hidden gaugino is heavier than the lightest neutralino, $M_{X}>M_{\chi_{1}^{0}}$. Firstly, due to the mixing with the bino, the interactions of the hidden gaugino are automatically "bino-like". Secondly, for the small mixings that we consider bounds from overproduction arguments are irrelevant [30]. Note that the results from this paragraph can also hold in that case, provided one exchanges the roles of the hidden gaugino and the lightest neutralino. However, this requires that all sparticles, apart from the lightest neutralino, are heavier than the hidden gaugino. Generically this will not be the case and the hidden gaugino will cascade-decay through the different sparticles down into the lightest neutralino. We will consider this in detail in the next subsection.

\subsection{Decaying hidden gauginos}

A hidden gaugino that is heavier than the lightest neutralino, $M_{X}>M_{\chi_{1}^{0}}$, turns out to be more appealing from the phenomenological point of view. In this case, the mass of the lightest neutralino can be small and of the order of a few $100 \mathrm{GeV}$, and the hidden gaugino automatically possesses the "bino-like" interactions which are desirable for the leptophilic decay. We will again assume that the lightest neutralino makes up most of the dark matter, whereas the hidden gaugino contributes only a subdominant part $\rho_{X} \ll \rho_{\chi_{1}^{0}}$ to the overall matter density of the Universe. Note that in this case the lifetime of the hidden gaugino can be as small as $\tau_{X} \sim 10^{17} \mathrm{~s}$, the current age of the Universe, provided that its relic abundance is small enough. For definiteness, we will take $\rho_{X}=10^{-3} \rho_{\chi_{1}^{0}}$ throughout this section.

We will firstly discuss the contributions from the decaying hidden gaugino to the cosmicray flux as predicted for our reference mSUGRA scenario. Secondly we will consider the multi-peak structure of a cascade-decaying hidden gaugino in light of the ATIC data.

mSUGRA point. The considerations in this paragraph are again based on the mSUGRA scenario described above. Depending on the mass of the hidden gaugino, its decay can produce fermions, neutralinos, charginos, Higgs and gauge bosons as depicted in table 1. The corresponding branching ratios are summarized in table 5, where we do not show the subsequent decays of the neutralinos $\chi_{2,3,4}^{0}$ and charginos $\chi_{1,2}^{ \pm}$for simplicity. ${ }^{12}$

As apparent from table 5 , the decay into charged lepton/slepton pairs is dominant in the whole mass range $M_{X} \simeq 600-1600 \mathrm{GeV}$ that we consider. The decay into quarks is suppressed by the large squark masses, $m_{\tilde{q}} \gtrsim 1.1 \mathrm{TeV}$, whereas decay into $h^{0}, Z^{0}$ and $W^{ \pm}$bosons is mainly suppressed by the small mixing between higgsinos and the hidden

\footnotetext{
${ }^{11}$ Winos only couple to left-handed sleptons, which are typically heavier than the right-handed ones, whereas higgsinos can easily decay into the Higgs boson.

${ }^{12}$ These subsequent decays are taken into account in our calculations. We singled out the dominant decay modes in our reference mSUGRA model and used them in the PYTHIA code: $X \rightarrow h^{0} \chi_{4}^{0}, X \rightarrow Z^{0} \chi_{3}^{0}$, $X \rightarrow W^{\mp} \chi_{2}^{ \pm}, \chi_{3}^{0} \rightarrow \chi_{1}^{0} Z^{0}, \chi_{4}^{0} \rightarrow \chi_{1}^{0} h^{0}$ and $\chi_{2}^{ \pm} \rightarrow \chi_{1}^{ \pm} Z^{0}(28 \%), \chi_{1}^{ \pm} h^{0}(27 \%), \chi_{2}^{0} W^{ \pm}(36 \%)$. The decay of $\chi_{2}^{0}$ and $\chi_{1}^{ \pm}$only produces leptons and is neglected.
} 


\begin{tabular}{|cccccccc|}
\hline \multirow{2}{*}{$M_{X}[\mathrm{GeV}]$} & $\nu \tilde{\nu}$ & \multicolumn{1}{c}{ Branching Ratios for $X \rightarrow$} & $q \tilde{q}$ & $h^{0} \chi_{i}^{0}$ & $Z^{0} \chi_{i}^{0}$ & $W^{ \pm} \chi_{i}^{\mp}$ & \multirow{2}{*}{$\tau_{X}\left[10^{23} \mathrm{~s}\right]$} \\
\hline 600 & $1.8 \%$ & $98.2 \%$ & - & $0.1 \%$ & $0.0 \%$ & - & 1.1 \\
700 & $5.6 \%$ & $92.9 \%$ & - & $0.6 \%$ & $0.0 \%$ & $0.9 \%$ & \\
800 & $5.6 \%$ & $84.6 \%$ & - & $3.5 \%$ & $0.2 \%$ & $6.1 \%$ & 1.0 \\
850 & $0.7 \%$ & $49.8 \%$ & - & $17.3 \%$ & $1.2 \%$ & $31.0 \%$ & \\
900 & $15.3 \%$ & $53.7 \%$ & - & $10.7 \%$ & $0.9 \%$ & $19.4 \%$ & 0.8 \\
1000 & $14.1 \%$ & $81.1 \%$ & - & $1.4 \%$ & $1.0 \%$ & $2.4 \%$ & \\
1200 & $13.3 \%$ & $76.8 \%$ & - & $2.7 \%$ & $2.5 \%$ & $4.7 \%$ & 0.7 \\
1400 & $13.2 \%$ & $74.1 \%$ & $1.6 \%$ & $2.9 \%$ & $2.8 \%$ & $5.4 \%$ & \\
1600 & $12.5 \%$ & $68.5 \%$ & $8.4 \%$ & $2.7 \%$ & $2.7 \%$ & $5.2 \%$ & \\
\hline
\end{tabular}

Table 5. Branching ratios of the dominant decay modes of a hidden gaugino that is cascade-decaying into the MSSM particle zoo. The underlying scenario is our chosen mSUGRA reference point as described in the text. The decay into neutrinos and charged leptons is essentially democratic in all three flavors. We also indicate the lifetime of the hidden gaugino that gives the best fit to the PAMELA data, assuming an energy density of $\rho_{X}=10^{-3} \rho_{\mathrm{DM}}$.
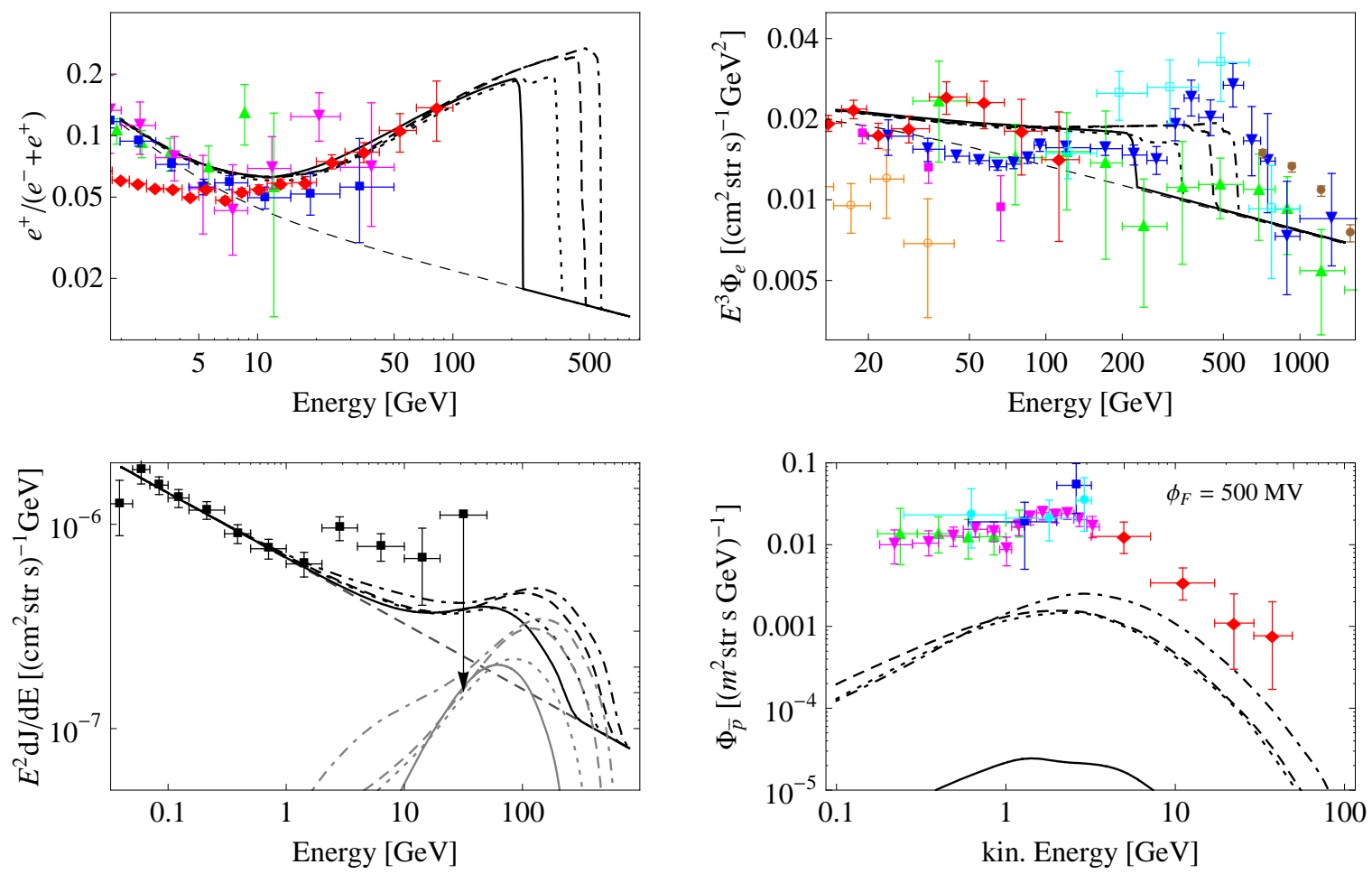

Figure 3. Positron fraction, extragalactic gamma-ray flux, antiproton flux and total electron + positron flux from the decay of a hidden gaugino as predicted by our mSUGRA scenario. The branching ratios are shown in table 5. The mass of the hidden gaugino varies between $600 \mathrm{GeV}$ (solid), $800 \mathrm{GeV}$ (dotted), $1000 \mathrm{GeV}$ (dashed) and $1200 \mathrm{GeV}$ (dot-dashed).

gaugino. However, this mixing can become enhanced when the masses of the higgsinos become comparable to the mass of the hidden gaugino, which happens around $M_{X} \sim 870 \mathrm{GeV}$.

Our results for the cosmic-ray fluxes are shown in figure 3 for hidden gaugino masses 


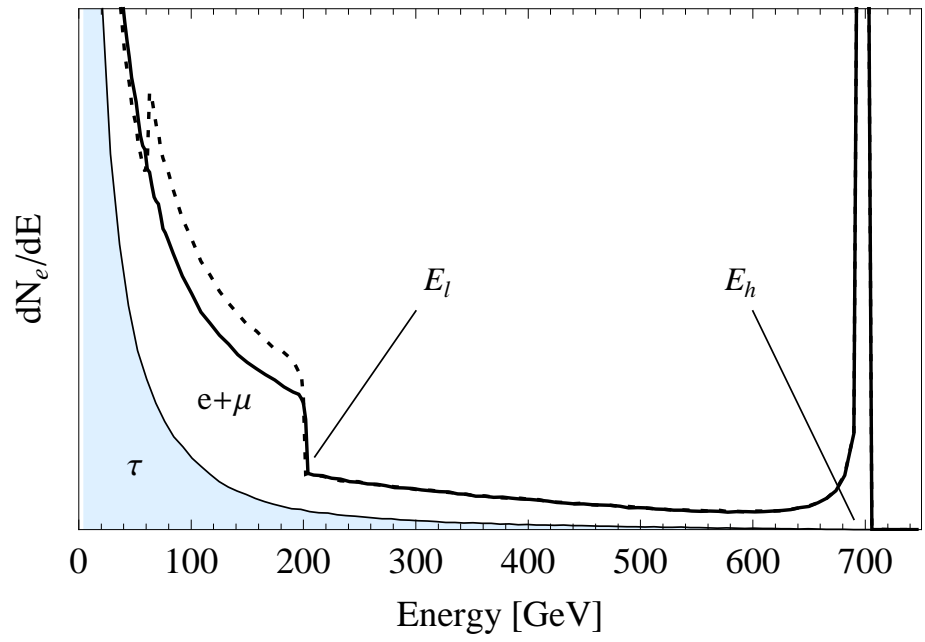

Figure 4. Energy spectrum of positrons from an idealized cascade-decaying hidden gaugino. Only two-body decay into right-handed slepton/lepton pairs is taken into account. The slepton subsequently decays into the lightest neutralino. The spectrum exhibits two pronounced peaks, which we denote by $E_{h}$ and $E_{l}$. We show plots for a lightest neutralino with mass $150 \mathrm{GeV}$ (solid) and with $1 \mathrm{TeV}$ (dashed). The position of the peaks is fixed to $E_{h}=700 \mathrm{GeV}$ and $E_{l}=200 \mathrm{GeV}$, as suggested by the ATIC data. The masses of the right-handed sleptons follow then from eq. (4.1). We also indicate the part of the positrons that comes solely from the tau/stau decay channel (blue area).

between $600 \mathrm{GeV}$ and $1200 \mathrm{GeV}$, where we adjusted the lifetime of the hidden gaugino to fit the PAMELA data. For all masses of the hidden gaugino that we consider, the predictions for the positron fraction are in qualitatively good agreement with the PAMELA data. At the same time, the contribution to the antiproton flux lies well below the measurements and hence is safe in all cases. Furthermore, we obtain contributions to the extragalactic gamma-ray flux, which are mainly due to $\tau$ decays. They are compatible with the EGRET measurements but could show up in future experiments as an excess above background. The total electron+positron flux is also compatible with the different measurements and we predict a sharp step at high energies.

Multi-peak structures from cascade decays. As already evident in figure 3, the energy distribution of particles produced in cascade decays in general features several peaks. Their exact position carries information about the masses of the different intermediate particles. It is intriguing to speculate that the apparent double-peak structure of the ATIC data originates from cascade-decaying particles [12]. ${ }^{13}$

In the case of the decaying hidden gaugino, the energy spectrum of positrons in general possesses two pronounced peaks. These peaks stem from decays with intermediate selectrons. To simplify the discussion, we will neglect decay modes that produce $h^{0}, Z^{0}$ and $W^{ \pm}$bosons or left-handed sleptons, and we will assume democratic decay into all three flavors. If we furthermore assume approximate mass degeneracy for the three right-handed sleptons, we are left with only three free parameters: the mass of the hidden gaugino $M_{X}$, the mass of the lightest neutralino $M_{\chi_{1}^{0}}$, and the mass scale of the right-handed sleptons $M_{\tilde{l}_{R}}$.

In figure 4 we show the corresponding energy spectrum of positrons for two different

\footnotetext{
${ }^{13}$ Note, however, that the recently released electron data from the Fermi satellite [55] does not confirm the double peak structure seen by ATIC. For a discussion about the implications of the new data for our model see the appendix. Other explanations for double-peak structures can be found in ref. [60].
} 

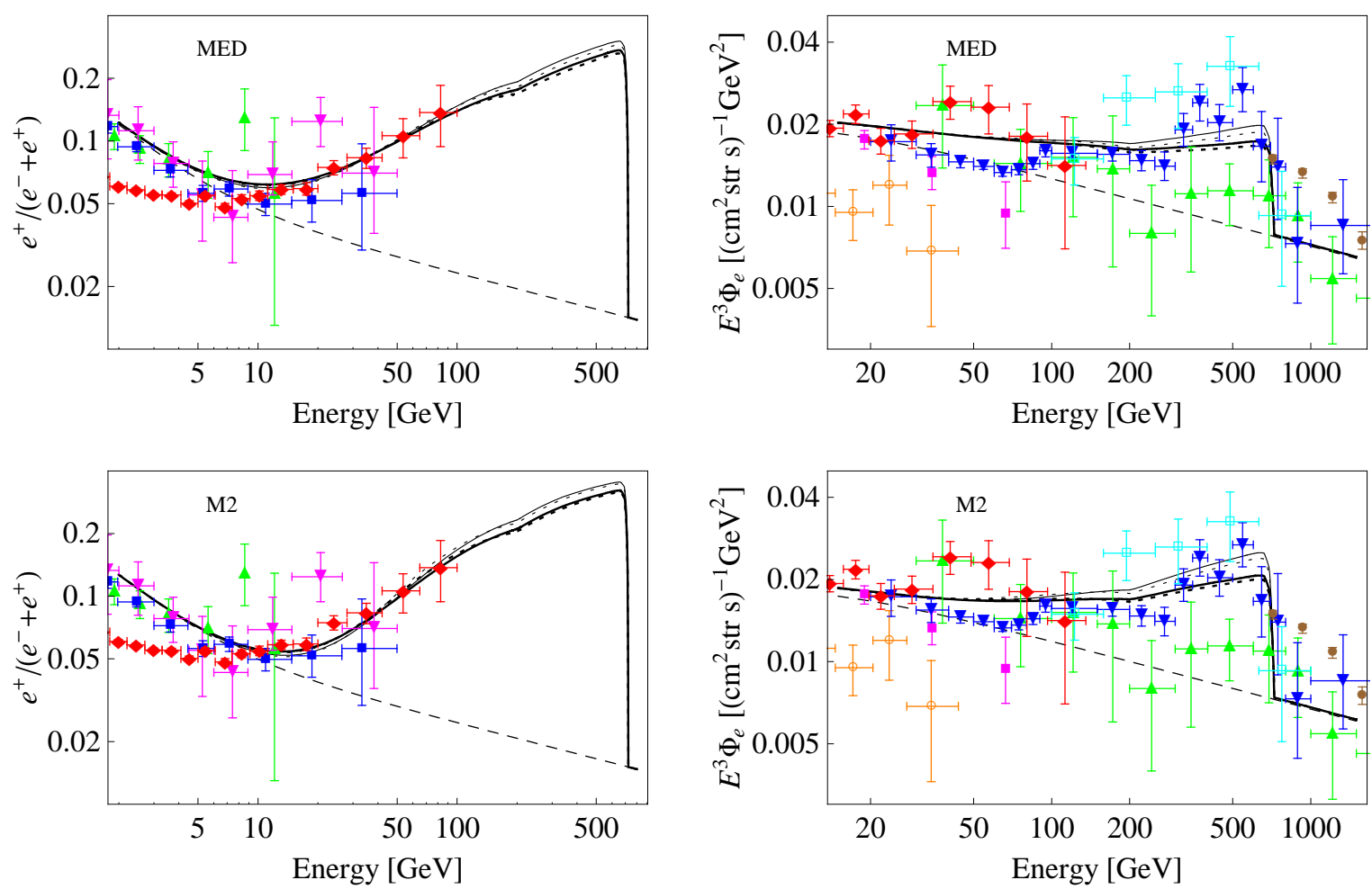

Figure 5. Positron fraction and total electron+positron flux for an idealized cascade-decaying hidden gaugino. Like in figure 4, only leptonic decay modes are taken into account. We assume democratic decay into the three right-handed charged sleptons (thick lines), or into only the selectron and smuon (thin lines). The mass of the lightest neutralino varies between $150 \mathrm{GeV}$ (solid) and $1 \mathrm{TeV}$ (dotted). We show the plots for the propagation models MED (upper plots) and M2 (lower plots) of ref. [47] (see table 2).

sets of particle masses. The two pronounced peaks are denoted by $E_{h}$ and $E_{l}$. Fixing the neutralino mass and the position of the peaks determines the slepton and hidden gaugino masses according to

$$
\begin{aligned}
& M_{\tilde{l}_{R}}^{2}=M_{\chi_{1}^{0}}^{2}+2 E_{l}^{2}\left(\sqrt{\left(\frac{E_{h}}{E_{l}}-1\right)^{2}+\left(\frac{M_{\chi_{1}^{0}}}{E_{l}}\right)^{2}}-\frac{E_{h}}{E_{l}}+1\right), \\
& M_{X}=E_{h}+\sqrt{E_{h}^{2}+M_{\tilde{l}_{R}}^{2}} .
\end{aligned}
$$

As a simple attempt to fit the ATIC data with an idealized cascade-decaying hidden gaugino, we take the values $E_{h}=700 \mathrm{GeV}$ and $E_{l}=200 \mathrm{GeV}$. After this, a neutralino mass of $M_{\chi_{1}^{0}}=150 \mathrm{GeV}(1000 \mathrm{GeV})$ implies a slepton mass of $M_{\tilde{l}_{R}}=177 \mathrm{GeV}(1117 \mathrm{GeV})$ and a hidden gaugino mass of $M_{X}=1422 \mathrm{GeV}(2018 \mathrm{GeV})$.

The resulting cosmic-ray fluxes for the two neutralino masses are shown in figure 5 (upper plots, thick lines), where we used the MED propagation model. Interestingly, the predicted fluxes are practically the same for the two cases, although the bump structure is slightly enhanced in case of the heavier neutralino. The lower plots are based on the M2 propagation model as an exemplary model with a thin diffusion zone, $L=1 \mathrm{kpc}$ (as opposed to $L=4 \mathrm{kpc}$ in case of the MED model). Since in these scenarios electrons and positrons are 
more likely to escape the diffusion zone before having lost much of their energy, the observable spectrum becomes steeper. This effect can improve the agreement with the sharp features of the ATIC data. However, note that propagation models with thin diffusion zones have problems with the correct prediction of the flux of unstable isotopes like ${ }^{10} \mathrm{Be}$ or ${ }^{14} \mathrm{C}$ [61]. ${ }^{14}$

Up to now we have assumed a vanishing stau mixing angle and democratic decay into all three flavors. However, if the stau mixing angle is large the decay mode into tau/stau pairs would be suppressed, as discussed above. In figure 4 we indicated the part of the positrons that comes from the stau/tau channel in the case of democratic decay (blue region). A suppression of this channel can lead to a relative enhancement of the two-peak structure of the cascade decay. This effect is shown by the thin lines in figure 5, where we only took into account the decay modes into muon/smuon and electron/selectron pairs. As expected, the peaks at high energy become more pronounced, and the spectrum becomes harder at low energies, although the effect is not dramatic.

\section{Conclusions}

In this work we have shown that a simple extension of the MSSM by an additional hidden Abelian gauge group $\mathrm{U}(1)_{\mathrm{X}}$, which kinetically mixes with the hypercharge $\mathrm{U}(1)_{\mathrm{Y}}$, can account for the observed PAMELA excess if the kinetic mixing parameter is in the range of $\chi \sim 10^{-(20 \ldots 24)}$. We also briefly discussed possible origins of such a tiny mixing in scenarios with warped extra dimensions. Depending on the masses, either the visible-sector neutralino or the hidden gaugino becomes unstable and subject to decay. We have demonstrated that this decay is dominated by leptonic modes in certain parameter regions of the MSSM where the sleptons are light (see table 4 and table 5 ). We found that a decaying hidden gaugino with a mass around $600-1200 \mathrm{GeV}$ can naturally explain the observed excess in the positron fraction without overproducing antiprotons (see figure 3). Furthermore, we showed that it is difficult to accommodate a sharp double-peak structure as observed by ATIC within our setup and with standard propagation models. Our considerations suggest a preference for supersymmetric models with relatively light sleptons, as e.g. realized in mSUGRA models which lie in the coannihilation region. In any case, we predict a contribution to the extragalactic gamma-ray flux, which mainly stems from tau decays, and which should be observable by future experiments like the Fermi Gamma-ray Space Telescope.

\section{Acknowledgments}

We would like to thank A. Strong for providing a convenient compilation of cosmic-ray data. Furthermore, CW gratefully acknowledges G. Sigl for helpful discussions and the Technische Universität München for kind hospitality. AI and DT would like to thank the Yukawa Institute for Theoretical Physics and the CERN Theory Division for hospitality during the last stages of this work. The work of AI and DT was partially supported by the DFG cluster of excellence "Origin and Structure of the Universe."

During the last stages of our work a preprint appeared that also examines hidden gauginos in the context of the PAMELA/ATIC anomalies, coming to similar conclusions [63].

\footnotetext{
${ }^{14}$ Furthermore, the change of the propagation model in principle also changes the predictions for the background of secondary positrons, which were calculated for the above M1, M2 and MED model in ref. [62]. However, in figure 5 we used the background from ref. [2] since the backgrounds obtained in ref. [62] are meant to be extreme cases.
} 

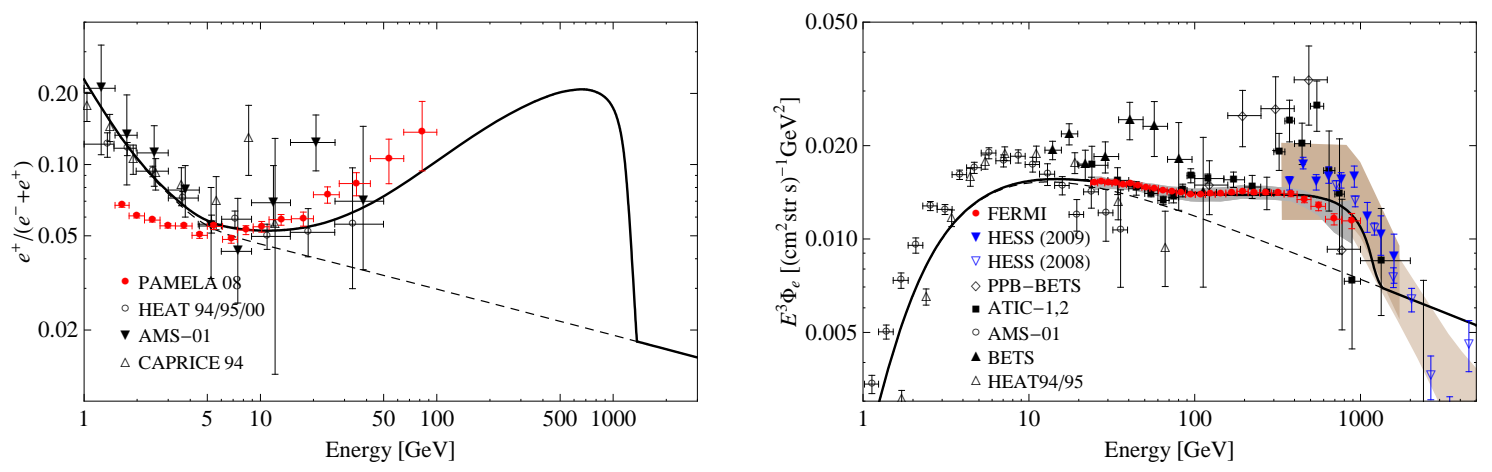

Figure 6. Positron fraction and total electron+positron flux for an idealized, three-body decaying bino-like neutralino with mass $2500 \mathrm{GeV}$, decaying into a light hidden gaugino. We also show the new Fermi LAT [55] and H.E.S.S. [64] data points.

\section{A Implications of recent Fermi LAT results}

The recently released measurement of the cosmic-ray electron+positron spectrum from the Fermi LAT [55] does not confirm the distinct feature seen by the ATIC experiment. Instead, Fermi LAT observes a smooth, hard spectrum, which follows a power law $\sim E^{-3.0}$ up to energies of $1 \mathrm{TeV}$. As indicated by the H.E.S.S. observations [64], this power law further steepens at energies above $1 \mathrm{TeV}$.

In figure 6 we show the results for a three-body decaying bino-like neutralino, ${ }^{15}$ as discussed in section 4.1. The mass of the neutralino is $2500 \mathrm{GeV}$, whereas the hidden gaugino has a small mass $\sim \mathcal{O}(100 \mathrm{GeV})$. In the plots we assume that the decay is only mediated by right-handed sleptons with a mass close to the neutralino mass. All other supersymmetric particles must be heavy enough to make their effects on the branching ratios negligible. However, although this scenario is phenomenologically appealing, it requires some non-standard cosmology in order to avoid the thermal overproduction of the heavy bino-like neutralino. A second way to explain the data measured by Fermi would be a cascade-decaying hidden gaugino, see section 4.2. The sharp step in the electron+positron spectrum, as shown in figure 3, disappears if the first generation sleptons are heavy enough to suppress the production of electrons or positrons in two-body decays. This, however, requires non-universal slepton masses.

Note that in both of the above cases, the production and decay of taus will lead to a visible bump in the extragalactic gamma-ray flux, which should be measurable by the Fermi LAT.

\section{References}

[1] PAMELA collaboration, O. Adriani et al., An anomalous positron abundance in cosmic rays with energies 1.5-100 GeV, Nature 458 (2009) 607 [arXiv:0810.4995] [SPIRES].

[2] I.V. Moskalenko and A.W. Strong, Production and propagation of cosmic-ray positrons and electrons, Astrophys. J. 493 (1998) 694 [astro-ph/9710124] [SPIRES].

[3] J.J. Beatty et al., New measurement of the cosmic-ray positron fraction from 5 GeV to 15 GeV, Phys. Rev. Lett. 93 (2004) 241102 [astro-ph/0412230] [SPIRES].

[4] M. Boezio et al., The cosmic-ray electron and positron spectra measured at 1 AU during solar minimum activity, Astrophys. J. 532 (2000) 653.

\footnotetext{
${ }^{15}$ For further details about the plot see ref. [65].
} 
[5] AMS-01 collaboration, M. Aguilar et al., Cosmic-ray positron fraction measurement from $1 \mathrm{GeV}$ to $30 \mathrm{GeV}$ with AMS-01, Phys. Lett. B 646 (2007) 145 [astro-ph/0703154] [SPIRES].

[6] A.J. Tylka, Cosmic ray positrons from annihilation of weakly interacting massive particles in the galaxy, Phys. Rev. Lett. 63 (1989) 840 [SPIRES];

M.S. Turner and F. Wilczek, Positron line radiation from halo WIMP annihilations as a dark matter signature, Phys. Rev. D 42 (1990) 1001 [SPIRES];

M. Kamionkowski and M.S. Turner, A Distinctive positron feature from heavy WIMP annihilations in the galactic halo, Phys. Rev. D 43 (1991) 1774 [SPIRES];

G.L. Kane, L.-T. Wang and J.D. Wells, Supersymmetry and the positron excess in cosmic rays, Phys. Rev. D 65 (2002) 057701 [hep-ph/0108138] [SPIRES];

E.A. Baltz, J. Edsjo, K. Freese and P. Gondolo, The cosmic ray positron excess and neutralino dark matter, Phys. Rev. D 65 (2002) 063511 [astro-ph/0109318] [SPIRES];

G.L. Kane, L.-T. Wang and T.T. Wang, Supersymmetry and the cosmic ray positron excess, Phys. Lett. B 536 (2002) 263 [hep-ph/0202156] [SPIRES];

H.-C. Cheng, J.L. Feng and K.T. Matchev, Kaluza-Klein dark matter,

Phys. Rev. Lett. 89 (2002) 211301 [hep-ph/0207125] [SPIRES];

D. Hooper and G.D. Kribs, Kaluza-Klein dark matter and the positron excess,

Phys. Rev. D 70 (2004) 115004 [hep-ph/0406026] [SPIRES];

M. Cirelli, R. Franceschini and A. Strumia, Minimal dark matter predictions for galactic positrons, anti-protons, photons, Nucl. Phys. B 800 (2008) 204 [arXiv:0802.3378] [SPIRES]; E.J. Chun and J.-C. Park, Dark matter and sub-GeV hidden U(1) in GMSB models, JCAP 02 (2009) 026 [arXiv:0812.0308] [SPIRES].

[7] E.A. Baltz and J. Edsjo, Positron propagation and fluxes from neutralino annihilation in the halo, Phys. Rev. D 59 (1998) 023511 [astro-ph/9808243] [SPIRES].

[8] J. Hisano, S. Matsumoto, O. Saito and M. Senami, Heavy wino-like neutralino dark matter annihilation into antiparticles, Phys. Rev. D 73 (2006) 055004 [hep-ph/0511118] [SPIRES].

[9] L. Bergstrom, T. Bringmann and J. Edsjo, New positron spectral features from supersymmetric dark matter - a way to explain the PAMELA data?, Phys. Rev. D 78 (2008) 103520 [arXiv:0808.3725] [SPIRES];

V. Barger, W.Y. Keung, D. Marfatia and G. Shaughnessy, PAMELA and dark matter, Phys. Lett. B 672 (2009) 141 [arXiv:0809.0162] [SPIRES];

I. Cholis, D.P. Finkbeiner, L. Goodenough and N. Weiner, The PAMELA positron excess from annihilations into a light boson, arXiv:0810.5344 [SPIRES];

D. Feldman, Z. Liu and P. Nath, PAMELA positron excess as a signal from the hidden sector, Phys. Rev. D 79 (2009) 063509 [arXiv:0810.5762] [SPIRES];

P.J. Fox and E. Poppitz, Leptophilic dark matter, Phys. Rev. D 79 (2009) 083528 [arXiv:0811.0399] [SPIRES];

I. Cholis, G. Dobler, D.P. Finkbeiner, L. Goodenough and N. Weiner, The case for a $700+$ gev WIMP: cosmic Ray Spectra from ATIC and PAMELA, arXiv:0811.3641 [SPIRES].

[10] A. Ibarra and D. Tran, Antimatter signatures of gravitino dark matter decay, JCAP 07 (2008) 002 [arXiv:0804.4596] [SPIRES].

[11] K. Ishiwata, S. Matsumoto and T. Moroi, High energy cosmic rays from the decay of gravitino dark matter, Phys. Rev. D 78 (2008) 063505 [arXiv:0805.1133] [SPIRES];

C.-R. Chen, F. Takahashi and T.T. Yanagida, Gamma rays and positrons from a decaying hidden gauge boson, Phys. Lett. B 671 (2009) 71 [arXiv:0809.0792] [SPIRES];

C.-R. Chen and F. Takahashi, Cosmic rays from leptonic dark matter, JCAP 02 (2009) 004 [arXiv:0810.4110] [SPIRES];

P.-f. Yin et al., PAMELA data and leptonically decaying dark matter,

Phys. Rev. D 79 (2009) 023512 [arXiv:0811.0176] [SPIRES];

K. Ishiwata, S. Matsumoto and T. Moroi, Cosmic-ray positron from superparticle dark matter and the PAMELA anomaly, Phys. Lett. B 675 (2009) 446 [arXiv:0811.0250] [SPIRES];

C.-R. Chen, M.M. Nojiri, F. Takahashi and T.T. Yanagida, Decaying hidden gauge boson and the PAMELA and ATIC/PPB-BETS anomalies, arXiv:0811.3357 [SPIRES]; 
E. Nardi, F. Sannino and A. Strumia, Decaying dark matter can explain the electron/positron excesses, JCAP 01 (2009) 043 [arXiv:0811.4153] [SPIRES];

K. Hamaguchi, S. Shirai and T.T. Yanagida, Cosmic ray positron and electron excess from hidden-fermion dark matter decays, Phys. Lett. B 673 (2009) 247 [arXiv:0812.2374] [SPIRES];

M. Pospelov and M. Trott, R-parity preserving super-WIMP decays, JHEP 04 (2009) 044 [arXiv: 0812.0432] [SPIRES].

[12] A. Arvanitaki et al., Astrophysical probes of unification, arXiv:0812.2075 [SPIRES].

[13] M. Cirelli, M. Kadastik, M. Raidal and A. Strumia, Model-independent implications of the $e^{ \pm}$, $\bar{p}$ cosmic ray spectra on properties of Dark Matter, Nucl. Phys. B $\mathbf{8 1 3}$ (2009) 1 [arXiv: 0809.2409] [SPIRES].

[14] A. Ibarra and D. Tran, Decaying dark matter and the PAMELA anomaly, JCAP 02 (2009) 021 [arXiv:0811.1555] [SPIRES].

[15] A.W. Strong, I.V. Moskalenko and O. Reimer, A new determination of the extragalactic diffuse gamma-ray background from EGRET data, Astrophys. J. 613 (2004) 956 [astro-ph/0405441] [SPIRES].

[16] R. Essig, N. Sehgal and L.E. Strigari, Bounds on cross-sections and lifetimes for dark matter annihilation and decay into charged leptons from gamma-ray observations of dwarf galaxies, Phys. Rev. D 80 (2009) 023506 [arXiv:0902.4750] [SPIRES].

[17] O. Adriani et al., A new measurement of the antiproton-to-proton flux ratio up to $100 \mathrm{GeV}$ in the cosmic radiation, Phys. Rev. Lett. 102 (2009) 051101 [arXiv:0810.4994] [SPIRES].

[18] H. Matsunaga et al., Measurement of low-energy cosmic-ray antiprotons at solar minimum, Phys. Rev. Lett. 81 (1998) 4052 [astro-ph/9809326] [SPIRES].

[19] BESS collaboration, S. Orito et al., Precision measurement of cosmic-ray antiproton spectrum, Phys. Rev. Lett. 84 (2000) 1078 [astro-ph/9906426] [SPIRES].

[20] WIZARD collaboration, M. Boezio et al., The cosmic-ray antiproton flux between 0.62 GeV and $3.19 \mathrm{GeV}$ measured near solar minimum activity, Astrophys. J. 487 (1997) 415 [SPIRES].

[21] WiZARD/CAPRICE collaboration, M. Boezio et al., The cosmic-ray anti-proton flux between $3 \mathrm{GeV}$ and $49 \mathrm{GeV}$, Astrophys. J. 561 (2001) 787 [astro-ph/0103513] [SPIRES].

[22] J.W. Mitchell et al., Measurement of $0.25 \mathrm{GeV}$ to 3.2 GeV anti-protons in the cosmic radiation, Phys. Rev. Lett. 76 (1996) 3057 [SPIRES].

[23] J. Chang et al., An excess of cosmic ray electrons at energies of 300-800 GeV, Nature 456 (2008) 362 [SPIRES].

[24] M.A. DuVernois et al., Cosmic ray electrons and positrons from $1 \mathrm{GeV}$ to $100 \mathrm{GeV}$ : measurements with HEAT and their interpretation, Astrophys. J. 559 (2001) 296 [SPIRES].

[25] PPB-BETS collaboration, S. Torii et al., High-energy electron observations by PPB-BETS flight in Antarctica, arXiv:0809.0760 [SPIRES].

[26] H.E.S.S. collaboration, F. Aharonian et al., The energy spectrum of cosmic-ray electrons at TeV energies, Phys. Rev. Lett. 101 (2008) 261104 [arXiv:0811.3894] [SPIRES].

[27] S. Torii et al., The energy spectrum of cosmic ray electrons from $10 \mathrm{GeV}$ to $100 \mathrm{GeV}$ observed with a highly granulated imaging calorimeter, Astrophys. J. 559 (2001) 973 [SPIRES].

[28] T. Kobayashi et al., High energy cosmic-ray electrons beyond $100 \mathrm{GeV}$, prepared for $26^{\text {th }}$ International Cosmic Ray Conference (ICRC 1999), Salt Lake City, U.S.A., 17-25 Aug 1999 [SPIRES].

[29] A.K. Harding and R. Ramaty, The pulsar contribution to galactic cosmic ray positrons, Proc. of the 20 ${ }^{\text {th }}$ International Cosmic Ray Conference (ICRC 1987), Moscow, Russia, 1987, pg. 92; 
A.M. Atoian, F.A. Aharonian and H.J. Volk, Electrons and positrons in the galactic cosmic rays, Phys. Rev. D 52 (1995) 3265 [SPIRES];

X. Chi, E.C.M. Young and K.S. Cheng, Pulsar-wind origin of cosmic ray positrons, Astrophys.

J. 459 (1995) L83 [SPIRES];

C. Grimani, Pulsar birthrate set by cosmic-ray positron observations,

Astron. Astrophys. 418 (2004) 649 [SPIRES];

D. Hooper, P. Blasi and P.D. Serpico, Pulsars as the sources of high energy cosmic ray positrons, JCAP 01 (2009) 025 [arXiv:0810.1527] [SPIRES].

[30] A. Ibarra, A. Ringwald and C. Weniger, Hidden gauginos of an unbroken U(1): cosmological constraints and phenomenological prospects, JCAP 01 (2009) 003 [arXiv:0809.3196] [SPIRES].

[31] B. Holdom, Two U(1)'s and epsilon charge shifts, Phys. Lett. B 166 (1986) 196 [SPIRES].

[32] J.L. Feng, H. Tu and H.-B. Yu, Thermal relics in hidden sectors, JCAP 10 (2008) 043 [arXiv:0808.2318] [SPIRES].

[33] L. Randall and R. Sundrum, Out of this world supersymmetry breaking, Nucl. Phys. B 557 (1999) 79 [hep-th/9810155] [SPIRES].

[34] O. Lebedev et al., A mini-landscape of exact MSSM spectra in heterotic orbifolds, Phys. Lett. B 645 (2007) 88 [hep-th/0611095] [SPIRES].

[35] S.A. Abel, J. Jaeckel, V.V. Khoze and A. Ringwald, Illuminating the hidden sector of string theory by shining light through a magnetic field, Phys. Lett. B 666 (2008) 66 [hep-ph/0608248] [SPIRES].

[36] C.-R. Chen, F. Takahashi and T.T. Yanagida, High-energy Cosmic-Ray Positrons from Hidden-Gauge-Boson Dark Matter, Phys. Lett. B 673 (2009) 255 [arXiv:0811.0477] [SPIRES].

[37] K.R. Dienes, C.F. Kolda and J. March-Russell, Kinetic mixing and the supersymmetric gauge hierarchy, Nucl. Phys. B 492 (1997) 104 [hep-ph/9610479] [SPIRES].

[38] S.A. Abel and B.W. Schofield, Brane-antibrane kinetic mixing, millicharged particles and SUSY breaking, Nucl. Phys. B 685 (2004) 150 [hep-th/0311051] [SPIRES].

[39] S.A. Abel, M.D. Goodsell, J. Jaeckel, V.V. Khoze and A. Ringwald, Kinetic mixing of the photon with hidden $U(1) s$ in string phenomenology, JHEP 07 (2008) 124 [arXiv:0803.1449] [SPIRES].

[40] J.P. Conlon, F. Quevedo and K. Suruliz, Large-volume flux compactifications: moduli spectrum and D3/D7 soft supersymmetry breaking, JHEP 08 (2005) 007 [hep-th/0505076] [SPIRES].

[41] S. Kachru, R. Kallosh, A. Linde and S.P. Trivedi, de Sitter vacua in string theory, Phys. Rev. D 68 (2003) 046005 [hep-th/0301240] [SPIRES].

[42] L. Covi, M. Grefe, A. Ibarra and D. Tran, Unstable gravitino dark matter and neutrino flux, JCAP 01 (2009) 029 [arXiv:0809.5030] [SPIRES].

[43] G. Bertone, W. Buchmüller, L. Covi and A. Ibarra, Gamma-rays from decaying dark matter, JCAP 11 (2007) 003 [arXiv:0709.2299] [SPIRES];

A. Ibarra and D. Tran, Gamma ray spectrum from gravitino dark matter decay, Phys. Rev. Lett. 100 (2008) 061301 [arXiv:0709.4593] [SPIRES].

[44] J.F. Navarro, C.S. Frenk and S.D.M. White, The structure of cold dark matter halos, Astrophys. J. 462 (1996) 563 [astro-ph/9508025] [SPIRES].

[45] V.L. Ginzburg, V.A. Dogiel, V.S. Berezinsky, S.V. Bulanov and V.S. Ptuskin, Astrophysics of cosmic rays, North-Holland, Amsterdam The Netherlands (1990), pg. 534.

[46] D. Maurin, F. Donato, R. Taillet and P. Salati, Cosmic Rays below $Z=30$ in a diffusion model: new constraints on propagation parameters, Astrophys. J. 555 (2001) 585 [astro-ph/0101231] [SPIRES]. 
[47] T. Delahaye, R. Lineros, F. Donato, N. Fornengo and P. Salati, Positrons from dark matter annihilation in the galactic halo: theoretical uncertainties, Phys. Rev. D 77 (2008) 063527 [arXiv:0712.2312] [SPIRES].

[48] F. Donato, N. Fornengo, D. Maurin and P. Salati, Antiprotons in cosmic rays from neutralino annihilation, Phys. Rev. D 69 (2004) 063501 [astro-ph/0306207] [SPIRES].

[49] L. Zhang, G. Sigl and J. Redondo, Galactic signatures of decaying dark matter, arXiv:0905.4952 [SPIRES].

[50] L.C. Tan and L.K. Ng, Calculation of the equilibrium anti-proton spectrum, J. Phys. G 9 (1983) 227 [SPIRES].

[51] F. Donato et al., Antiprotons from spallation of cosmic rays on interstellar matter, Astrophys. J. 563 (2001) 172 [astro-ph/0103150] [SPIRES].

[52] L.J. Gleeson and W.I. Axford, Cosmic rays in the interplanetary medium, Astrophys. J. 149 (1967) L115.

[53] L.J. Gleeson and W.I. Axford, Solar modulation of galactic cosmic rays, Astrophys. J. 154 (1968) 1011.

[54] J.S. Perko, Solar modulation of galactic antiprotons, Astron. Astrophys. 184 (1987) 119.

[55] TheFermi LAT collaboration, A.A. Abdo et al., Measurement of the cosmic ray $e^{+}+e^{-}$ spectrum from $20 \mathrm{GeV}$ to $1 \mathrm{TeV}$ with the Fermi Large Area Telescope, Phys. Rev. Lett. 102 (2009) 181101 [arXiv:0905.0025] [SPIRES].

[56] P. Gondolo et al., Dark SUSY: computing supersymmetric dark matter properties numerically, JCAP 07 (2004) 008 [astro-ph/0406204] [SPIRES]; see http://www.physto.se/ edsjo/darksusy.

[57] T. Hahn, Generating Feynman diagrams and amplitudes with FeynArts 3, Comput. Phys. Commun. 140 (2001) 418 [hep-ph/0012260] [SPIRES].

[58] T. Hahn and M. Pérez-Victoria, Automatized one-loop calculations in four and D dimensions, Comput. Phys. Commun. 118 (1999) 153 [hep-ph/9807565] [SPIRES].

[59] T. Sjöstrand, S. Mrenna and P. Skands, PYTHIA 6.4 Physics and Manual, JHEP 05 (2006) 026 [hep-ph/0603175] [SPIRES].

[60] K. Cheung, P.-Y. Tseng and T.-C. Yuan, Double-action dark matter, PAMELA and ATIC, arXiv:0902.4035 [SPIRES].

[61] A.W. Strong, I.V. Moskalenko and V.S. Ptuskin, Cosmic-ray propagation and interactions in the Galaxy, Ann. Rev. Nucl. Part. Sci. 57 (2007) 285 [astro-ph/0701517] [SPIRES].

[62] T. Delahaye et al., Galactic secondary positron flux at the Earth, arXiv:0809.5268 [SPIRES].

[63] S. Shirai, F. Takahashi and T.T. Yanagida, Decaying hidden gaugino as a source of PAMELA/ATIC anomalies, arXiv:0902.4770 [SPIRES].

[64] H.E.S.S. collaboration, F. Aharonian, Probing the ATIC peak in the cosmic-ray electron spectrum with H.E.S.S, arXiv:0905.0105 [SPIRES].

[65] A. Ibarra, D. Tran and C. Weniger, Decaying dark matter in light of the PAMELA and Fermi LAT data, arXiv:0906.1571 [SPIRES]. 